
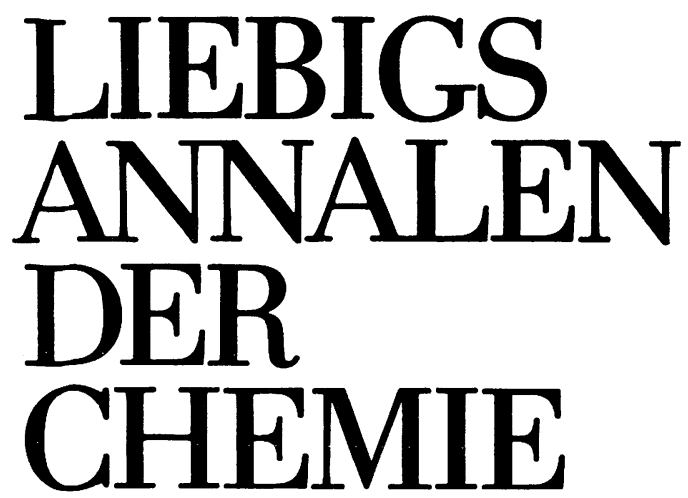

GEGRÜNDET 1832

JAHRGANG 1982

HERAUSGEGEBEN IM AUFTRAG DER

GESELLSCHAFT DEUTSCHER CHEMIKER

VON

B. FRANCK - K. HAFNER - H. MUSSO - H. PAULSEN - E. SCHMITZ

U. SCHÖLLKOPF · K. SCHREIBER · H. A. STAAB · TH. WIELAND

REDAKTION: H. ZAHN

mit W. JUNG und R. TEMME

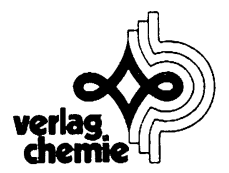




\section{AUTORENREGISTER}

Banerjee, S. K., 699

Bußmann, W., 805

Echter, T., 683

Engels, J., 745

Ernst, L., 651

Fleischmann, K., 675

Franke, A., 794

Frey, M., 817

Fritz, H., 739

Gnichtel, H., 729

Götze, A., 805

Gupta, B. D., 699

Heyns, K., 780

Höfle, G., 699

Huth, H.-U., 739

Huttner, G., 745

Jäger, V., 817

Jiang Xiang-jun, 717

John, H.-G., 613, 626, 639

Kazimierczuk, Z., 754

Knappe, W. R., 762

Köll, P., 613, 626, 639

Kopf, J., 626, 639

Krahmer, U., 745

Kresze, G., 723
Meier, H., 683

Mengel, R., 651

Menkhoff, S., 813

Merkle, U., 683

Meyer, B., 780

Morr, M., 651, 666

Pérez, M. A., 723

Pfleiderer, W., 754, 762

Ram, V. J., 762

Redlich, H., 708, 717

Roedig, A., 675

Röper, H., 780

Röper, S., 780

Rössert, M., 723

Salem, W. I., 729

Schulz, J., 613

Schwöbel, A., 723

Seela, F., 805, 813

Sheldrick, W. S., 699

Vogel, T., 739

Wannagat, U., 734

Wrobel, D., 734

Zimmer, O., 683

Zsolnai, L., 745 


\title{
Eine einfache neue Synthese der Fusarinsäure und anderer 5-Alkyl-2-pyridincarbonsäuren
}

\author{
Elke Langhals, Heinz Langhals und Christoph Rüchardt* \\ Chemisches Laboratorium der Universität Freiburg, \\ Albertstr. 21, D-7800 Freiburg i. Br.
}

Eingegangen am 9. November 1981

In 3-Acylpyridine läßt sich durch Minisci-Reaktion mit Formamiden, Fe(II)-sulfat und tert-Butylhydroperoxid gezielt in die 6-Position eine Carboxamidgruppe einführen. Die Aufarbeitung wird durch Zugabe von Citronensäure als Komplexbildner für Eisenionen wesentlich verbessert. Durch anschließende Wolff-Kishner-Reduktion erhält man direkt Fusarinsäure und andere 5-Alkyl-2pyridincarbonsäuren in $20-75 \%$ Ausbeute über beide Schritte.

\section{A New Simple Synthesis of Fusaric Acid and Other 5-Alkyl-2-pyridinecarboxylic Acids}

Carboxamido groups are introduced into the 6-position of 3-acylpyridines with high regioselectivity via Minisci reaction with formamides, Fe(II) sulfate and tert-butyl hydroperoxide. The isolation procedure is considerably improved by the addition of citric acid for complexation of the Fe ions. Fusaric acid and other 5-alkyl-2-pyridine carboxylic acids are obtained by a following Wolff-Kishner reaction in $20-75 \%$ overall yield.

Eine Reihe pharmakologisch wirksamer Substanzen leitet sich von 2,5-disubstituierten Pyridinen mit jeweils zwei unterschiedlichen Substituenten ab, so z. B. die Fusarinsäure (1) und andere 5-substituierte 2-Pyridincarbonsäuren 2, deren blutdrucksenkende Wirkung bekannt ist ${ }^{1-4)}$.<smiles>CCCCc1ccc(C(=O)O)nc1</smiles><smiles>[R]c1ccc(C(=O)O)nc1</smiles>

Verbindungen mit diesem Substitutionsmuster sind jedoch schwer zugänglich ${ }^{4)}$. So ist man bisher darauf angewiesen, entweder den Pyridinring aus geeignet substituierten Bausteinen durch Kondensationsreaktionen aufzubauen ${ }^{5)}$, oder an leichter zugänglichen 2,5-Dialkylpyridinen eine gezielte Funktionalisierung vorzunehmen ${ }^{6-9)}$.

Der zunächst naheliegende und einfachste Weg, in 3-Stellung substituierte Pyridine durch Substitution gezielt in 1 zu überführen, wurde bisher nur in einem Fall erfolgreich beschritten ${ }^{10}$ ) und steht z.Zt. nicht als allgemeine Synthesemethode zur Verfügung. Elektrophile greifen 3-Acylpyridine im allgemeinen in 5-Position an, Nucleophile wie z. B. metallorganische Reagenzien dagegen in 2-, 4- oder 6-Position, zum Teil auch mehrfach ${ }^{11)}$. Nur durch Emmert-Reaktion ${ }^{12,13)}$ von 3-Butylpyridin mit amalgamiertem Magnesium und sterisch anspruchsvollen Ketonen wurde die gewünschte Substitution in 6-Position erreicht und durch weitere Oxidation Fusarinsäure in mäßi-

(C) Verlag Chemie GmbH, D-6940 Weinheim, 1982 
ger Ausbeute zugänglich ${ }^{10}$ ). Dieses Syntheseprinzip wurde bisher noch nicht verallgemeinert. Da bei diesen Verfahren eine Verunreinigung des Produktes durch Quecksilberorganyle nur schwer auszuschließen ist ${ }^{10}$ ), ist es für die Herstellung der pharmakologisch wirksamen Verbindungen 1 und $2^{1-4,8,14)}$ nur begrenzt brauchbar.

Der nach Arbeiten von Minisci ${ }^{15-17)}$ aussichtsreich erscheinende Weg, zur Synthese von 2-Vertretern 3-Alkylpyridiniumsalze radikalisch zu acylieren, in der Hoffnung, durch den sterischen Effekt von R bevorzugt Angriff in 6-Stellung zu erreichen, konnte nicht realisiert werden.

Einmal lagen die Ausbeuten bei der Minisci-Acylierung einfacher Alkylpyridine wie $\beta$ - und $\gamma$-Picolin, 4-Ethyl- oder 3-Butylpyridin mit Aldehyden, DMF oder Trioxan und tert-Butylhydroperoxid/Fe(II)-sulfat als Redoxinitiator bei höchstens $15 \%$ und teilweise stark darunter ${ }^{18}$. Zum anderen traten dabei eine Reihe von Nebenprodukten auf, die teilweise auf radikalische Substitution in Benzylstellung, teilweise auf die bekannte Mehrfachacylierung zurückzuführen sind ${ }^{15-17)}$. Der eingeführte erste Acylrest fördert bekanntlich durch seine elektronenanziehende Wirkung ${ }^{19)}$ die Zweitsubstitution durch nucleophile Acylradikale ${ }^{15-17)}$.

So erhielt man bei der Umsetzung von 4-Ethylpyridin in DMF mit 1 Äquivalent $\mathrm{H}_{2} \mathrm{SO}_{4}$, tert-Butylhydroperoxid und $\mathrm{Fe}(\mathrm{II})$-sulfat bei ca. $12 \%$ Umsatz nach GC-MSAnalyse die in Schema 1 aufgeführten Substitutionsprodukte ${ }^{18)}$.
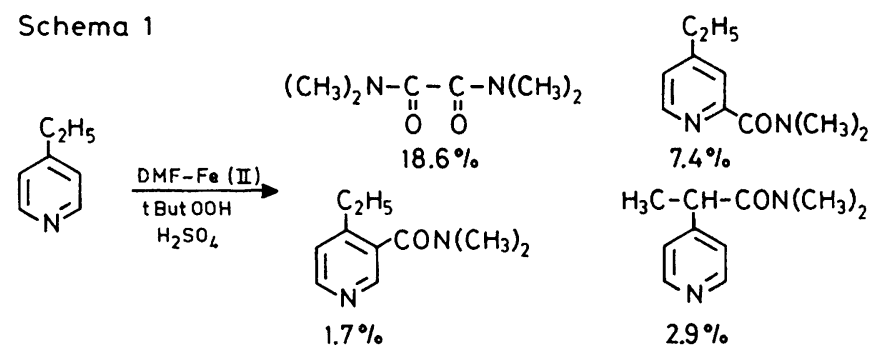

Über die Bildung von Tetramethyloxamid wurde bereits früher berichtet ${ }^{20)}$. Neu ist der Befund, daß Dimethylcarbamoylierung auch in 3-Stellung und in Benzylposition eintritt. Letztere kann durch Radikaldimerisation oder durch Angriff des „Benzylradikals“ an DMF selbst mit anschließender Dehydrierung zustandekommen. $\gamma$-Picolin lieferte mit Acetaldehyd und tert-Butoxyradikalen 10\% 2-Acetyl- und 2,6-Diacetyl- $\gamma$ picolin $^{18,20)}, \beta$-Picolin sogar bis zu 16\% 2,4,6-Triacetyl- $\beta$-picolin ${ }^{18,21)}$. Eine Steuerung des Ausbeuteverhältnisses durch den $\mathrm{pH}-$ Wert ${ }^{15-17)}$ gelang nicht ${ }^{18)}$. Bei dem Versuch, 3-Butylpyridin mit Pivalaldehyd nach Minisci ${ }^{17)}$ umzusetzen, entstand unter Decarbonylierung 2-tert-Butyl-5-butylpyridin und als Nebenprodukte fand man u.a. 1-(3-Pyridyl)butanol und tert-Butyl-3-butyrylpyridin mit unbekanntem Substitutionsmuster.

Als besseres Synthesekonzept erwies sich die Carbamoylierung von 3-Acylpyridinen 3 mit DMF oder Formamid, da diese Substanzen erhöhte Reaktivität besitzen ${ }^{15-17)}$ und außerdem die störende Reaktivität in Benzylstellung fehlt.

Die benötigten 3-Acylpyridine 3 sind gut zugänglich. Friedel-Crafts-Acylierungen ${ }^{22}$ mit Nikotinsäurechlorid führen in guter Ausbeute zu Arylketonen. Die Umsetzung von 3-Cyanpyridin mit Grignard-Verbindungen liefert über die entsprechenden Ketimine

Liebigs Ann. Chem. 1982 
und deren Hydrolyse universell aliphatische oder aromatische 3-Acylpyridine ${ }^{23)}$. Die als Zwischenprodukte auftretenden Ketimine sind allerdings teilweise schwer hydrolysierbar. Umpolung der Aldehydgruppe des 3-Pyridincarbaldehyds und Michael-Addition an Acrylnitril nach Stetter ${ }^{24)}$ führt zu einem zusätzlich funktionalisierten 3-Acylpyridin. Schließlich läßt sich 3-Pyridincarbaldehyd auch zweistufig mit Grignard-Reagens und durch anschließende Oxidation ${ }^{25)}$ in die gewünschten 3-Acylpyridine überführen.

Als erstes sei die Synthese der Fusarinsäure(1) selbst aus 1-(3-Pyridyl)-1-butanon (3, $\mathrm{R}=\mathrm{C}_{3} \mathrm{H}_{7}$ ) besprochen. Die radikalische Substitution sollte so durchgeführt werden, daß sich die neu eingeführte Gruppe leicht in eine Carboxylgruppe überführen läßt. Besonders aussichtsreich hierfür schien die Synthese von 5-Butanoyl-2-pyridincarboxamiden 4 durch Umsetzung von $3\left(\mathrm{R}=\mathrm{C}_{3} \mathrm{H}_{7}\right)$ mit DMF, Formamid oder Monoalkylformamid, Peroxid und Eisen(II)-Salzen, die nach Minisci ${ }^{15-17,26)}$ unter milden Bedingungen eintreten soll. Dabei war zu hoffen, daß die Substitution von $3\left(\mathrm{R}=\mathrm{C}_{3} \mathrm{H}_{7}\right)$ wegen des sterischen Effektes des Acylrestes in 6-Stellung erfolgt.

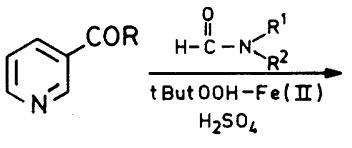

3

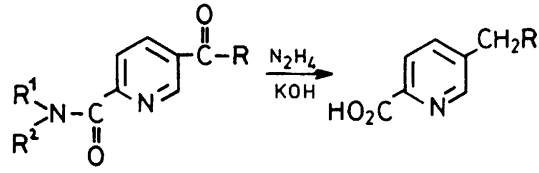

4

Die Reaktion von $3\left(\mathrm{R}=\mathrm{C}_{3} \mathrm{H}_{7}\right)$ mit DMF nach Minisci ergab zwar laut GC in der Tat das gewünschte Produkt $4\left(\mathrm{R}=\mathrm{C}_{3} \mathrm{H}_{7}, \mathrm{R}^{1}=\mathrm{R}^{2}=\mathrm{CH}_{3}\right)$, dessen Isolierung bereitete aber Schwierigkeiten. Aus saurem Medium läßt sich 4 naturgemäß nicht extrahieren. Im neutralen oder alkalischen Bereich fällt andererseits schwerlösliches Eisen(III)-oxidhydrat aus, welches das Reaktionsprodukt bindet. Die Isolierung gelang erst durch Komplexierung der Eisenionen mit Citronensäure ${ }^{18,27)}$. Unter Verwendung von DMF und Formamid selbst konnte dabei 4 in Ausbeuten von $20 \%\left(\mathrm{R}^{1}=\mathrm{R}^{2}=\mathrm{CH}_{3}\right)$ bzw. $31 \%\left(R^{1}=R^{2}=H\right)$ isoliert werden. Während die Reaktion mit DMF wegen vermutlich hochmolekularer Nebenprodukte chromatographisch aufgearbeitet werden muß, kann aus der Reaktionslösung der Formamidreaktion $4\left(R^{1}=R^{2}=H\right)$ durch einfache Extraktion mit $\mathrm{CHCl}_{3}$ oder durch Fällung mit Wasser als kristalline einheitliche Reinsubstanz isoliert werden. Das Substitutionsmuster wurde spektroskopisch und durch Überführung in Fusarinsäure selbst bestimmt. Am sichersten läßt sich das Substitutionsmuster aus der chemischen Verschiebung und der Größe der aromatischen Protonenkopplungskonstanten im ${ }^{1} \mathrm{H}$-NMR-Spektrum erkennen.

Bei der Umsetzung von $3\left(\mathrm{R}=\mathrm{C}_{3} \mathrm{H}_{7}\right)$ mit tert-Butylformamid entstand aus ungeklärtem Grund durch Substitution in 2- $(1 \%)$, 4- (3\%) und 6-Stellung (2\%) ein Isomerengemisch neben 3\% Doppelsubstitution in 4- und 6-Stellung von 3.

Zur Synthese der Fusarinsäure (1) wurde das Amid bzw. das Dimethylamid $4(\mathrm{R}=$ $\mathrm{C}_{3} \mathrm{H}_{7}$ ) der Wolff-Kishner-Reduktion ${ }^{23)}$ unterworfen. Dabei trat neben der Reduktion der Ketofunktion gleichzeitig Hydrolyse der Säureamidfunktion ein, so daß $\mathbf{1}$ aus 2 $\left(\mathrm{R}=\mathrm{COC}_{3} \mathrm{H}_{7}\right)$ in zwei Reaktionsschritten mit insgesamt $20 \%$ Ausbeute zugänglich ist. Dieses Verfahren zur Synthese von 1 kann damit gut mit den komplizierteren in der Literatur beschriebenen Synthesen konkurrieren. 
Tab. 1. Darstellung der 5-Acyl-2-pyridincarboxamide $4\left(R^{1}=R^{2}=H\right)$ durch radikalische Carbamoylierung von 3 mit $\mathrm{HCONH}_{2}$ und Wolff-Kishner-Reduktion zu 5-Alkyl-2-pyridincarbonsäuren 5

\begin{tabular}{rlcl}
\hline Nr. & \multicolumn{1}{c}{$\mathrm{R}$} & \% Ausb. 4 & \% Ausb. 5 \\
\hline 1 & $\mathrm{C}_{3} \mathrm{H}_{7}$ & 31 & 65 \\
2 & $\mathrm{C}_{6} \mathrm{H}_{5}$ & 45 & 75 \\
3 & $4-\mathrm{Cl}_{2}-\mathrm{C}_{6} \mathrm{H}_{4}$ & 80 & 92 \\
4 & $4-\mathrm{F}_{6} \mathrm{H}_{4}$ & 79 & a) \\
5 & $4-\mathrm{CH}_{3} \mathrm{O}_{-}-\mathrm{C}_{6} \mathrm{H}_{4}$ & 57 & 53 \\
6 & $4-\mathrm{CBu}_{6}-\mathrm{C}_{6} \mathrm{H}_{4}$ & 82 & 83 \\
7 & $4-\mathrm{CF}_{3}-\mathrm{C}_{6} \mathrm{H}_{4}$ & 52 & 45 c) \\
8 & $3-\mathrm{CF}_{3}-\mathrm{C}_{6} \mathrm{H}_{4}$ & 50 & 68 \\
9 & $2-\mathrm{Methylpropyl}_{1}$ & 20 & b) \\
10 & $2,2-\mathrm{Dimethylpropyl}$ & 27 & b) \\
11 & NC- $-\mathrm{CH}_{2}-\mathrm{CH}_{2}$ & 91 & 65 d) \\
\hline
\end{tabular}

a) Bei der Reduktion konnte noch kein einheitliches Produkt isoliert werden. - b) Reduktion wurde noch nicht durchgeführt. - c) Unter den Reaktionsbedingungen wird die Amidgruppe nicht verseift. - d) Unter gleichzeitiger Verseifung der Nitrilgruppe zur Carbonsäuregruppe.

Aus diesem Grund wurde auch die Verallgemeinerung dieses neuen Syntheseprinzips zur Darstellung anderer 5-Alkyl-2-pyridincarbonsäuren ausgearbeitet. Die Ergebnisse enthält Tab. 1, die eine recht allgemeine Anwendungsbreite der Synthese erkennen läßt. Die Carbamoylierung nach Minisci gelingt sowohl bei aliphatischen Ketonen (Nr. 1, 9, 10), bei funktionalisierten aliphatischen Ketonen (Nr. 11) als auch bei aromatischen Ketonen (Nr. 2-8). Sie versagte lediglich, wenn der aliphatische Rest R in 3 über ein tertiäres C-Atom an den Carbonylkohlenstoff gebunden ist wie in 3-Pyridylcyclopentyl- oder -cyclohexylketon. Es wurden dann bei geringem Umsatz vorwiegend höher molekulare Produkte festgestellt. Die Reduktion zu den 5-Alkyl-2-pyridincarbonsäuren 5 gelingt in fast allen Fällen in guter bis sehr guter Ausbeute. Eine zusätzliche Variation ergibt sich durch die Darstellung der in $m$ - oder $p$-Stellung nitrierten Derivate, die weitere Substitutionsvariationen wie üblich ermöglichen. Die bisher durchgeführten Syntheseschritte zeigt Schema 2.

Schema 2

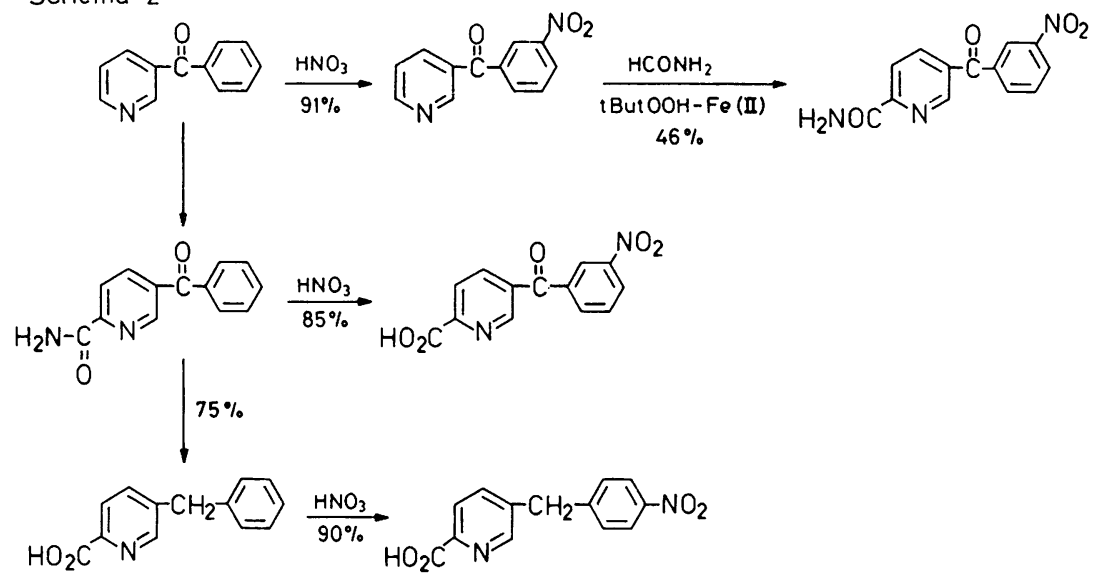


Für pharmakologische Anwendungen als Antihypertensiva sind sowohl die Fusarinsäure-Analogen 5 selbst als auch deren Vorstufen, die Carbonsäureamide 4, von Interesse $^{28)}$, die gegebenenfalls über den Metabolismus die freie Säure bilden.

Dem Fonds der Chemischen Industrie danken wir für die Förderung dieser Arbeit, Herrn Dr. D. Hunkler für die Aufnahme der NMR-Spektren sowie Herrn Dr. J. Wörth für die der Massenspektren.

\section{Experimenteller Teil}

Folgende Geräte wurden verwendet: IR-Spektrometer: PE 457 der Fa. Perkin-Elmer; Gaschromatograph: F 20 B (FID) der Fa. Perkin-Elmer; NMR-Spektrometer: EM 390 der Fa. Varian und WM 250 der Fa. Bruker (TMS als interner Standard); Massenspektrometer: MAT 312 der Fa. Varian; Brechungsindices: Abbé-Refraktometer der Fa. Zeiss; Schmelzpunkte: H.-Bock-Monoscop der Fa. Büchi und Apparatur nach Dr. Tottoli der Fa. Büchi.

3-Acylpyridine 3 durch Friedel-Crafts-Acylierung: Die Synthese erfolgte in Anlehnung an Lit. ${ }^{22)}$ durch Umsetzung von frisch dargestelltem 3-Pyridincarbonsäurechlorid mit wasserfreiem Aluminiumtrichlorid und den entsprechenden Aromaten. Bei der Acylierung von Benzol, Chlorbenzol und Fluorbenzol diente der Aromat selbst als Lösungsmittel. Die Acylierung von Anisol und tert-Butylbenzol wurden in dem entsprechenden Volumen 1,2-Dichlorethan, über Aluminiumoxid filtriert, als Lösungsmittel durchgeführt. Die Aromaten wurden in der dreifachen molaren Menge in bezug auf 3-Pyridincarbonsäurechlorid eingesetzt. Reaktionsbedingungen, Ausbeuten und physikalische Daten siehe Tab. 2, spektroskopische Daten siehe Tab. 3.

3-(3-Nitrobenzoyl)pyridin: $9.15 \mathrm{~g}(50.0 \mathrm{mmol}) 3$-Benzoylpyridin werden bei $0{ }^{\circ} \mathrm{C}$ portionsweise in eine zuvor bereitete Nitriermischung aus $10 \mathrm{ml} 100$ proz. $\mathrm{HNO}_{3}$ und $14 \mathrm{ml} \mathrm{konz} . \mathrm{H}_{2} \mathrm{SO}_{4}$ unter Rühren eingetragen. Anschließend rührt man noch $2 \mathrm{~h}$ bei Raumtemp., gießt dann auf etwa $300 \mathrm{~g}$ zerstoßenes Eis und stellt mit halbkonz. Natronlauge alkalisch. Man extrahiert dreimal mit je $100 \mathrm{ml}$ Chloroform, wäscht die vereinigten Chloroformextrakte mit $100 \mathrm{ml}$ Wasser und trocknet sie mit Magnesiumsulfat. Nach Abdestillieren des Lösungsmittels verbleibt ein Öl, welches beim Stehenlassen kristallisiert. Umkristallisation aus Ether liefert blaßgelbe Rhomboeder. Ausb. und physikalische Daten siehe Tab. 2, spektroskopische Daten siehe Tab. 3.

3-Acylpyridine 3 durch Umsetzung von 3-Cyanpyridin mit Grignard-Reagenzien: Die Synthesen wurden in Anlehnung an Lit. ${ }^{23)}$ durchgeführt. Zur Aufarbeitung gießt man die Reaktionslösung auf eine Mischung aus $500 \mathrm{ml}$ Eis/Wasser und ca. $200 \mathrm{~g}$ Ammoniumchlorid und säuert mit ca. $100 \mathrm{ml} \mathrm{konz}$. Salzsäure an. Man extrahiert dreimal mit je $100 \mathrm{ml}$ Ether; die Etherextrakte werden verworfen. Unter Eiskühlung wird die wäßrige Phase durch Zusatz von halbkonz. Natronlauge alkalisch gemacht und dreimal mit je etwa $150 \mathrm{ml}$ Ether extrahiert. Die Etherextrakte werden mit $100 \mathrm{ml}$ Wasser gewaschen, mit Magnesiumsulfat getrocknet und destilliert. Bei $90^{\circ} \mathrm{C} / 15$ Torr gewinnt man nicht umgesetztes 3-Cyanpyridin zurück, bei höherer Temp. destillieren die Ketone 3 im Gemisch mit etwa 30-80\% der entsprechenden Imine. Zur Imin-Hydrolyse versetzt man die Imin-Keton-Gemische mit ca. $250 \mathrm{ml}$ halbkonz. Salzsäure und kocht bis zur vollständigen Hydrolyse unter Rückfluß (3-5 d, IR-spektroskopische oder gaschromatographische Kontrolle). Die Ketone werden aus der mit halbkonz. Natronlauge alkalisch gemachten Wasserphase durch Etherextraktion (ca. dreimal $150 \mathrm{ml}$ ) erhalten. Die vereinigten Etherextrakte werden mit $100 \mathrm{ml}$ Wasser gewaschen, mit Magnesiumsulfat getrocknet und eingedampft. Die Ketone fraktioniert man mit einer 1.5 -m-Drehbandkolonne $(\varnothing \approx 8 \mathrm{~mm}$ ). Flüssige Ketone werden als Pikrate charakterisiert. Ausb. und physikalische Daten der dargestellten Ketone siehe Tab. 4, spektroskopische Daten siehe Tab. 5. 
Tab. 2. Aromatische 3-Acylpyridine 3 durch Friedel-Crafts-Acylierung mit 3-Pyridincarbonsäurechlorid

\begin{tabular}{|c|c|c|c|c|c|c|c|c|c|}
\hline \multirow{2}{*}{ Keton } & \multirow{2}{*}{$T$ a) } & \multirow{2}{*}{$t^{\mathrm{b})}$} & \multirow{2}{*}{$\begin{array}{c}\% \\
\text { Ausb.c) }\end{array}$} & \multirow{2}{*}{$\begin{array}{l}\text { Schmp. } \\
{\left[{ }^{\circ} \mathrm{C}\right]}\end{array}$} & \multirow{2}{*}{$\begin{array}{l}\text { Summenformel } \\
\text { (Molmasse) }\end{array}$} & & \multicolumn{3}{|c|}{ Analyse } \\
\hline & & & & & & & C & $\mathrm{H}$ & $N$ \\
\hline 3-Benzoylpyridin & 83 & $3-4$ & $96^{\mathrm{c})}$ & $38^{f)}\left(39^{29 l}\right)$ & - & & - & - & - \\
\hline 3-(4-Chlorbenzoyl)pyridin & 130 & 4 & 92 & $88^{\text {g) }}\left(90-91^{30)}\right)$ & - & & - & - & - \\
\hline $\begin{array}{l}\text { 3-(4-Methoxybenzoyl)- } \\
\text { pyridin }\end{array}$ & $80^{h, i, k)}$ & $0.3^{\prime)}$ & 30 & $96^{\text {g) }}\left(99^{29)}\right)$ & - & & - & - & - \\
\hline
\end{tabular}


Tab. 3. Spektroskopische Daten der über Friedel-Crafts-Acylierung dargestellten 3-Acylpyridine 3

\begin{tabular}{|c|c|c|c|c|c|}
\hline Keton & $\begin{array}{l}\text { IR }(\mathrm{KBr})^{\mathrm{a})} \\
{\left[\mathrm{cm}^{-1}\right]}\end{array}$ & $\begin{array}{c}{ }^{1} \mathrm{H}-\mathrm{NMR}\left(\mathrm{CDCl}_{3}\right)^{\mathrm{b})} \\
\delta \text {-Werte }\end{array}$ & $\begin{array}{r}{ }^{13} \mathrm{C}-\mathrm{NM} \\
\delta\end{array}$ & $\begin{array}{l}\left(\mathrm{CDCl}_{3}\right) \\
\text { erte }\end{array}$ & $\begin{array}{c}\mathrm{MS}(70 \mathrm{eV}) \\
m / e\end{array}$ \\
\hline 3-Benzoylpyridin & $\begin{array}{l}3060 \mathrm{w}, 1660 \mathrm{~s}, \\
1645 \mathrm{~s}, 1585 \mathrm{~s}\end{array}$ & $\begin{array}{l}7.37-7.77(\mathrm{~m}, 5 \mathrm{H} \text { und } \mathrm{H}-5) \\
8.02(\mathrm{dt}, \mathrm{H}-4), 8.73(\mathrm{~m}, \mathrm{H}-6) \\
8.91(\mathrm{~m}, \mathrm{H}-2)\end{array}$ & $\begin{array}{l}123.26 \\
129.96(2 \mathrm{C}) \\
133.14 \\
137.02 \\
152.76\end{array}$ & $\begin{array}{l}128.58(2 \mathrm{C}) \\
133.06 \\
136.77 \\
150.88 \\
194.60(\mathrm{CO})\end{array}$ & - \\
\hline $\begin{array}{l}\text { 3-(4-Chlorbenzoyl)- } \\
\text { pyridin }\end{array}$ & $\begin{array}{l}3100 \mathrm{w}, 3060 \mathrm{w} \\
1640 \mathrm{~s}, 1585 \mathrm{~s} \\
\text { (Schulter) }\end{array}$ & $\begin{array}{l}7.41(\mathrm{dd}, \mathrm{H}-5), 7.58(\mathrm{mc} \\
\left.\mathrm{AA}^{\prime} \mathrm{BB}^{\prime}-\mathrm{Typ}, 4 \mathrm{H}\right), 8.04(\mathrm{dt} \\
\text { H-4), } 8.76 \text { (dd, H-6), } 8.90 \\
(\mathrm{~d}, \mathrm{H}-2)\end{array}$ & $\begin{array}{l}123.47 \\
131.43(2 \mathrm{C}) \\
135.10 \\
139.76 \\
153.09\end{array}$ & $\begin{array}{l}129.04(2 \mathrm{C}) \\
132.92 \\
137.07 \\
150.82 \\
193.56\end{array}$ & $\begin{array}{l}217\left(21 \%, \mathrm{M}^{\oplus}\right), 182\left(100, \mathrm{M}^{\oplus}-\mathrm{Cl}\right) \\
139\left(82, \mathrm{M}^{\oplus}-\mathrm{C}_{5} \mathrm{H}_{4} \mathrm{~N}\right), 111(30 \\
139-\mathrm{CO}), 106\left(29, \mathrm{C}_{5} \mathrm{H}_{4} \mathrm{NCO}^{\oplus}\right) \\
78\left(28, \mathrm{C}_{5} \mathrm{H}_{4} \mathrm{~N}^{\oplus}\right)\end{array}$ \\
\hline $\begin{array}{l}\text { 3-(4-Fluorbenzoyl)- } \\
\text { pyridinc) }\end{array}$ & $\begin{array}{l}3050 \mathrm{w}, 1655 \mathrm{~s}, \\
1600 \mathrm{~s}, 1585 \mathrm{~s}\end{array}$ & $\begin{array}{l}7.19 \text { und } 7.85\left(\mathrm{~m}, \mathrm{AA}^{\prime} \mathrm{BB}^{\prime} \mathrm{X}-\right. \\
\text { Typ, } 4 \mathrm{H}), 7.46(\mathrm{dd}, \mathrm{H}-5) \\
8.10(\mathrm{dt}, \mathrm{H}-4), 8.83(\mathrm{dd}, \\
\mathrm{H}-6), 8.96(\mathrm{~d}, \mathrm{H}-2)\end{array}$ & $\begin{array}{l}115.94\left(\mathrm{~d}, J_{\mathrm{C}}\right. \\
2 \mathrm{C}), 123.47, \\
J_{\mathrm{CF}}=9 \mathrm{~Hz}, \\
133.15\left(\mathrm{~d}, J_{\mathrm{C}}\right. \\
137.05,150.7 \\
165.85\left(\mathrm{~d}, J_{\mathrm{C}}\right. \\
216.08(\mathrm{CO})\end{array}$ & $\begin{array}{l}=22 \mathrm{~Hz} \\
2.74(\mathrm{~d} \\
\mathrm{C}), 132.84 \\
=2 \mathrm{~Hz}) \\
152.98 \\
=255 \mathrm{~Hz})\end{array}$ & - \\
\hline $\begin{array}{l}\text { 3-(4-Methoxy- } \\
\text { benzoyl)pyridin }\end{array}$ & $\begin{array}{l}3060 \mathrm{w}, 3040 \mathrm{w}, \\
2990 \mathrm{w}, 2950 \mathrm{w} \\
1645 \mathrm{~s}, 1600 \mathrm{~s} \\
1590 \mathrm{~s}\end{array}$ & $\begin{array}{l}3.82(\mathrm{~s}, 3 \mathrm{H}), 7.31(\mathrm{mc} \\
\left.\mathrm{AA}^{\prime} \mathrm{BB}^{\prime}-\mathrm{Typ}, 4 \mathrm{H}\right), 7.35(\mathrm{dd}, \\
\mathrm{H}-5), 8.00(\mathrm{dt}, \mathrm{H}-4), 8.70 \\
(\mathrm{dd}, \mathrm{H}-6), 8.87(\mathrm{~d}, \mathrm{H}-2)\end{array}$ & $\begin{array}{l}55.56 \\
123.29 \\
132.51(2 \mathrm{C}) \\
136.92 \\
152.41 \\
193.40(\mathrm{CO})\end{array}$ & $\begin{array}{l}113.93(2 \mathrm{C}) \\
129.46 \\
133.91 \\
150.55 \\
163.81\end{array}$ & $\begin{array}{l}213\left(42 \%, \mathrm{M}^{\oplus}\right), 198\left(11, \mathrm{M}^{\oplus}-\mathrm{CH}_{3}\right), \\
182\left(15, \mathrm{M}^{\oplus}-\mathrm{OCH}_{3}\right), 135(100, \\
\left.\mathrm{M}^{\oplus}-\mathrm{C}_{5} \mathrm{H}_{4} \mathrm{~N}\right), 107(15,135-\mathrm{CO})\end{array}$ \\
\hline $\begin{array}{l}\text { 3-(4-tert-Butyl- } \\
\text { benzoyl)pyridin }\end{array}$ & $\begin{array}{l}3050 \mathrm{w}, 3030 \mathrm{w} \\
2960 \mathrm{~m}, 1645 \mathrm{~s} \\
1605 \mathrm{~m}, 1580 \mathrm{~m}\end{array}$ & $\begin{array}{l}1.36(\mathrm{~s}, 9 \mathrm{H}), 7.45(\mathrm{dd}, \mathrm{H}-5) \\
7.65\left(\mathrm{mc}, \mathrm{AA}^{\prime} \mathrm{BB}^{\prime}-\mathrm{Typ}, 4 \mathrm{H}\right) \\
8.12(\mathrm{dt}, \mathrm{H}-4), 8.80(\mathrm{dd}, \\
\mathrm{H}-6), 9.02(\mathrm{~d}, \mathrm{H}-2)\end{array}$ & $\begin{array}{l}31.13(3 \mathrm{C}) \\
123.24 \\
126.66 \\
133.59 \\
150.84 \\
157.06\end{array}$ & $\begin{array}{l}35.20 \\
125.29(2 \mathrm{C}) \\
130.11(2 \mathrm{C}) \\
137.04 \\
152.60 \\
194.34(\mathrm{CO})\end{array}$ & $\begin{array}{l}239\left(27 \%, \mathrm{M}^{\oplus}\right), 224(100 \\
\left.\mathrm{M}^{\oplus}-\mathrm{CH}_{3}\right), 183\left(35, \mathrm{M}^{\oplus}-\mathrm{C}_{4} \mathrm{H}_{8}\right), \\
106\left(41, \mathrm{C}_{5} \mathrm{H}_{4} \mathrm{NCO}^{\oplus}\right), 78(39, \\
\left.\mathrm{C}_{5} \mathrm{H}_{4} \mathrm{~N}^{\oplus}\right), 51(17,78-\mathrm{HCN})\end{array}$ \\
\hline $\begin{array}{l}\text { 3-(3-Nitrobenzoyl)- } \\
\text { pyridin d) }\end{array}$ & $\begin{array}{l}3080 \mathrm{w}, 1655 \mathrm{~s}, \\
1610 \mathrm{~m}, 1585 \mathrm{~s}\end{array}$ & $\begin{array}{l}7.53(\mathrm{dd}, \mathrm{H}-5), 7.76(\mathrm{t} \\
\left.J=8 \mathrm{~Hz}, \mathrm{H}-5^{\prime}\right), 8.16(\mathrm{mc}, \\
\left.\mathrm{H}-4^{\prime} \text { und } \mathrm{H}-6^{\prime}\right), 8.49(\mathrm{dt} \\
\mathrm{H}-4), 8.63\left(\mathrm{mc}, \mathrm{H}-2^{\prime}\right), 8.86 \\
(\mathrm{dd}, \mathrm{H}-6), 9.00(\mathrm{~d}, \mathrm{H}-2)\end{array}$ & $\begin{array}{l}123.60,124.5 \\
129.97,131.9 \\
137.01,138.1 \\
150.72,153.6\end{array}$ & $\begin{array}{l}127.25 \\
135.24 \\
148.28 \\
192.40(\mathrm{CO})\end{array}$ & $\begin{array}{l}228\left(54 \%, \mathrm{M}^{\oplus}\right), 211\left(43, \mathrm{M}^{\oplus}-\mathrm{OH}\right) \\
150\left(55, \mathrm{M}^{\oplus}-\mathrm{C}_{5} \mathrm{H}_{4} \mathrm{~N}\right), 106(65 \\
\left.\mathrm{C}_{5} \mathrm{H}_{4} \mathrm{NCO}^{\oplus}\right), 78\left(100, \mathrm{C}_{5} \mathrm{H}_{4} \mathrm{~N}^{\oplus}\right) \\
51(87,78-\mathrm{HCN})\end{array}$ \\
\hline
\end{tabular}

a) Charakteristische Banden. $-{ }^{6}$ ) Alle Spektren werden nach 1. Ordnung interpretiert; ungefähre Kopplungskonstanten zwischen Pyridinprotonen: $J_{54}=$ $8 \mathrm{~Hz}, J_{65}=4-5 \mathrm{~Hz}, J_{64}=J_{42}=2 \mathrm{~Hz}$. - c) ${ }^{19} \mathrm{~F}-\mathrm{NMR}\left(\mathrm{CHCl}_{3}\right): \delta=-29(\mathrm{mc})$ bezogen auf Trifluoressigsäure-ethylester. - d) Durch Nitrierung; siehe 
Tab. 4. Darstellung von 3-Acylpyridinen 3

\begin{tabular}{|c|c|c|c|c|c|c|c|c|c|}
\hline Keton & Ausb. $a, b)$ & $\mathrm{Sdp} . / p^{\mathrm{b}, \mathrm{c}, \mathrm{d})}$ & $\left.n_{\mathrm{D}}^{20} \mathrm{~b}\right)$ & Schmp. ${ }^{c)}$ & $\begin{array}{l}\text { Summenformel } \\
\text { (Molmasse) }\end{array}$ & & $\mathrm{C}^{\mathrm{A}}$ & $\begin{array}{c}\text { Analyse } \\
\mathrm{H}\end{array}$ & $\mathrm{e}$ \\
\hline 1-(3-Pyridyl)-1-butanon ${ }^{f}$ & $24\left(40^{23)}\right)$ & $\begin{array}{l}120 / 15 \\
\left(94-98 / 3^{23)}\right)\end{array}$ & $\begin{array}{l}1.5134 \\
\left(1.5136^{23)}\right)\end{array}$ & - & - & & - & - & - \\
\hline $\begin{array}{l}\text { 3-Methyl-1-(3-pyridyl)- } \\
\text { 1-butanon })\end{array}$ & 20 & $134 / 15$ & 1.5086 & $49 / 106^{e)}$ & $\begin{array}{l}\mathrm{C}_{16} \mathrm{H}_{16} \mathrm{~N}_{4} \mathrm{O}_{8} \\
\quad(392.3)^{\mathrm{e})}\end{array}$ & $\begin{array}{l}\text { Ber.e) } \\
\text { Gef. }\end{array}$ & $\begin{array}{l}48.98 \\
48.95\end{array}$ & $\begin{array}{l}4.11 \\
4.04\end{array}$ & $\begin{array}{l}14.28 \\
13.65\end{array}$ \\
\hline $\begin{array}{l}\text { 3,3-Dimethyl-1-(3-pyridyl)- } \\
\text { 1-butanon f,g) }\end{array}$ & $5\left(25^{h)}\right)$ & $68 / 0.1$ & 1.5058 & $152^{e)}$ & $\begin{array}{l}\mathrm{C}_{17} \mathrm{H}_{18} \mathrm{~N}_{4} \mathrm{O}_{8} \\
\quad(406.4)^{\mathrm{e})}\end{array}$ & $\begin{array}{l}\text { Ber.e) } \\
\text { Gef. }\end{array}$ & $\begin{array}{l}50.25 \\
50.31\end{array}$ & $\begin{array}{l}4.47 \\
4.41\end{array}$ & $\begin{array}{l}13.79 \\
13.82\end{array}$ \\
\hline Cyclopentyl(3-pyridyl)keton ${ }^{\prime}$ & 26 & $146 / 15$ & 1.5412 & $115^{\mathrm{e})}$ & $\begin{array}{l}\mathrm{C}_{17} \mathrm{H}_{16} \mathrm{~N}_{4} \mathrm{O}_{8} \\
\quad(404.3)^{\mathrm{e})}\end{array}$ & $\begin{array}{l}\text { Ber.e) } \\
\text { Gef. }\end{array}$ & $\begin{array}{l}50.50 \\
50.52\end{array}$ & $\begin{array}{l}3.99 \\
4.07\end{array}$ & $\begin{array}{l}13.86 \\
13.98\end{array}$ \\
\hline $\begin{array}{l}\text { Cyclohexyl(3-pyridyl)- } \\
\text { keton }{ }^{\text {f }}\end{array}$ & 21 & $106 / 0.2$ & 1.5395 & $131 \mathrm{e})$ & $\begin{array}{l}\mathrm{C}_{18} \mathrm{H}_{18} \mathrm{~N}_{4} \mathrm{O}_{8} \\
\quad(418.4)^{\mathrm{e})}\end{array}$ & $\begin{array}{l}\text { Ber.e) } \\
\text { Gef. }\end{array}$ & $\begin{array}{l}51.68 \\
51.80\end{array}$ & $\begin{array}{l}4.34 \\
4.22\end{array}$ & $\begin{array}{l}13.39 \\
13.53\end{array}$ \\
\hline $\begin{array}{l}\text { 3-(4-Trifluormethyl)- } \\
\text { benzoyl)pyridin f) }\end{array}$ & 25 & $122 / 0.2$ & - & 54 & $\begin{array}{l}\mathrm{C}_{13} \mathrm{H}_{8} \mathrm{~F}_{3} \mathrm{NO} \\
(251.2)\end{array}$ & $\begin{array}{l}\text { Ber. } \\
\text { Gef. }\end{array}$ & $\begin{array}{l}62.16 \\
62.00\end{array}$ & $\begin{array}{l}3.21 \\
3.41\end{array}$ & $\begin{array}{l}5.58 \\
5.64\end{array}$ \\
\hline $\begin{array}{l}\text { 3-(3-Trifluormethylbenzoyl)- } \\
\left.\text { pyridin }{ }^{\circ}\right)\end{array}$ & 33 & - & - & $40 / 149 e)$ & $\begin{array}{l}\mathrm{C}_{19} \mathrm{H}_{11} \mathrm{~F}_{3} \mathrm{~N}_{4} \mathrm{O}_{8} \\
(480.3)^{\mathrm{e})}\end{array}$ & $\begin{array}{l}\text { Ber.e) } \\
\text { Gef. }\end{array}$ & $\begin{array}{l}47.51 \\
47.64\end{array}$ & $\begin{array}{l}2.31 \\
2.40\end{array}$ & $\begin{array}{l}11.66 \\
11.67\end{array}$ \\
\hline $\begin{array}{l}\text { 4-Oxo-4-(3-pyridyl)butan- } \\
\text { nitrili) }\end{array}$ & $80\left(78-84^{24)}\right)$ & $\begin{array}{l}149 / 0.1 \\
(150-152 / \\
\left.0.1^{24)}\right)\end{array}$ & - & $74\left(70-72^{24)}\right)$ & - & & - & - & - \\
\hline
\end{tabular}

a) Ausb. in \% . - b) Lit.-Werte in Klammern. - c) In ${ }^{\circ} \mathrm{C}$. - d) Druck in Torr. - e) Pikrat. - $n$ Durch Umsetzung von 3-Cyanpyridin mit den entsprechenden Grignard-Reagenzien. - g) Zweistufiger Syntheseweg (s.u.) ist vorzuziehen. - h) Zweistufiger Syntheseweg (s. u.). - i) Durch Umsetzung von 3-Pyridincarbaldehyd mit Acrylnitril siehe Lit. ${ }^{24)}$. 
Tab. 5. Spektroskopische Daten der Alkyl(3-pyridyl)ketone 3

\begin{tabular}{|c|c|c|c|c|}
\hline Keton & $\begin{array}{c}\mathrm{IR}^{\mathrm{a})} \\
{\left[\mathrm{cm}^{-1}\right]}\end{array}$ & $\begin{array}{c}\left.{ }^{1} \mathrm{H}-\mathrm{NMR}\left(\mathrm{CDCl}_{3}\right)^{\mathrm{b}}\right) \\
\delta \text {-Werte }\end{array}$ & 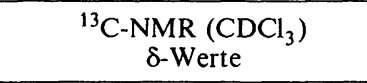 & $\begin{array}{c}\mathrm{MS}(70 \mathrm{eV}) \\
m / e\end{array}$ \\
\hline 1-(3-Pyridyl)-1-butanon & $\begin{array}{l}3030 \mathrm{w}, 2960 \mathrm{~s} \\
2870 \mathrm{~m}, 1765 \mathrm{~s} \\
1580 \mathrm{~s}\end{array}$ & $\begin{array}{l}1.02(\mathrm{t}, J=7 \mathrm{~Hz}, 3 \mathrm{H}) \\
1.78(\mathrm{sext}, J=7 \mathrm{~Hz}, 2 \mathrm{H}) \\
3.00(\mathrm{t}, J=7 \mathrm{~Hz}, 2 \mathrm{H}) \\
7.43(\mathrm{dd}, \mathrm{H}-5), 8.24(\mathrm{dd}, \\
\mathrm{H}-4), 8.77(\mathrm{dd}, \mathrm{H}-6), 9.10 \\
(\mathrm{~m}, \mathrm{H}-2)\end{array}$ & $\begin{array}{l}\text { 13.78, 17.42, 40.72, } 123.64 \\
132.25,135.24,149.64 \\
153.31,198.94(\mathrm{CO})\end{array}$ & $\begin{array}{l}149\left(28 \%, \mathrm{M}^{\oplus}\right), 134(5, \\
\left.\mathrm{M}^{\oplus}-\mathrm{CH}_{3}\right), 121(27, \\
\left.\mathrm{M}^{\oplus}-\mathrm{C}_{2} \mathrm{H}_{4}\right), 106(100, \\
\left.\mathrm{C}_{5} \mathrm{H}_{4} \mathrm{NCO}^{\oplus}\right), 78(92, \\
\left.\mathrm{M}^{\oplus}-\mathrm{C}_{3} \mathrm{H}_{7} \mathrm{CO}\right)\end{array}$ \\
\hline $\begin{array}{l}\text { 3-Methyl-1-(3-pyridyl)- } \\
\text { 1-butanon }\end{array}$ & $\begin{array}{l}3040 \mathrm{w}, 2950 \mathrm{~s} \\
2870 \mathrm{~m}, 1680 \mathrm{~s} \\
1580 \mathrm{~s}\end{array}$ & $\begin{array}{l}1.03(\mathrm{~d}, J=6 \mathrm{~Hz}, 6 \mathrm{H}), \\
2.23(\mathrm{mc}, 1 \mathrm{H}), 2.77(\mathrm{~d}, J= \\
6 \mathrm{~Hz}, 2 \mathrm{H}), 7.20(\mathrm{dd}, \mathrm{H}-5), \\
8.00(\mathrm{~m}, \mathrm{H}-4), 8.55(\mathrm{~m}, \\
\mathrm{H}-6), 8.97(\mathrm{~m}, \text { breit, H-2) }\end{array}$ & $\begin{array}{l}22.69(2 \mathrm{C}), 24.96,47.71 \\
123.65,132.58,135.37 \\
149.64,153.27,198.89(\mathrm{CO})\end{array}$ & $\begin{array}{l}163\left(31 \%, \mathrm{M}^{\oplus}\right), 148(15, \\
\left.\mathrm{M}^{\oplus}-\mathrm{CH}_{3}\right), 121(67, \\
\left.\mathrm{M}^{\oplus}-\mathrm{C}_{3} \mathrm{H}_{6}\right), 106(100, \\
\left.\mathrm{C}_{5} \mathrm{H}_{4} \mathrm{NCO}^{\oplus}\right), 78(63, \\
\left.\mathrm{C}_{5} \mathrm{H}_{4} \mathrm{~N}^{\oplus}\right), 57\left(7, \mathrm{C}_{4} \mathrm{H}_{9}^{\oplus}\right), \\
51(32,78-\mathrm{HCN}), 43(22, \\
\left.\mathrm{C}_{3} \mathrm{H}_{7}^{\oplus}\right)\end{array}$ \\
\hline $\begin{array}{l}\text { 3,3-Dimethyl-1-(3-pyridyl)- } \\
\text { 1-butanon }\end{array}$ & $\begin{array}{l}3040 \mathrm{w}, 2950 \mathrm{~s} \\
2900 \mathrm{~m}, 2860 \mathrm{~m} \\
1680 \mathrm{~s}, 1585 \mathrm{~s}\end{array}$ & $\begin{array}{l}1.05(\mathrm{~s}, 9 \mathrm{H}), 2.78(\mathrm{~s}, 2 \mathrm{H}) \\
7.25(\mathrm{dd}, \mathrm{H}-5), 8.08(\mathrm{dt}, \\
\mathrm{H}-4), 8.58(\mathrm{dd}, \mathrm{H}-6), 9.00 \\
(\mathrm{~d}, \mathrm{H}-2)^{\mathrm{c})}\end{array}$ & $\begin{array}{l}30.01(3 \mathrm{C}), 31.50,50.43 \\
123.55,133.70,135.41, \\
149.77,153.11,198.96(\mathrm{CO})\end{array}$ & - \\
\hline Cyclopentyl-(3-pyridyl)keton & $\begin{array}{l}3045 \mathrm{w}, 2960 \mathrm{~m} \\
2870 \mathrm{w}, 1685 \mathrm{~s} \\
1590 \mathrm{~s}\end{array}$ & $\begin{array}{l}1.83(\mathrm{~m}, 8 \mathrm{H}), 3.69 \text { (quint, } \\
J=8 \mathrm{~Hz}, 1 \mathrm{H}), 7.43(\mathrm{dd}, \\
\mathrm{H}-5), 8.25(\mathrm{dt}, \mathrm{H}-4), 8.76 \\
(\mathrm{dd}, \mathrm{H}-6), 9.13(\mathrm{~m}, \mathrm{H}-2)\end{array}$ & $\begin{array}{l}26.22(2 \mathrm{C}), 29.69(2 \mathrm{C}) \\
46.77,123.54,132.10 \\
135.71,149.98,153.09 \\
201.39(\mathrm{CO})\end{array}$ & $\begin{array}{l}175\left(35 \%, \mathrm{M}^{\oplus}\right), 147(18, \\
\left.\mathrm{M}^{\oplus}-\mathrm{C}_{2} \mathrm{H}_{4}\right), 134(61, \\
\left.\mathrm{M}^{\oplus}-\mathrm{C}_{3} \mathrm{H}_{5}\right), 106(100 \\
\left.\mathrm{C}_{5} \mathrm{H}_{4} \mathrm{NCO} \oplus\right), 78(52 \\
\left.\mathrm{C}_{5} \mathrm{H}_{4} \mathrm{~N} \mathrm{~N}^{\oplus}\right), 69\left(13, \mathrm{C}_{5} \mathrm{H}_{9}^{\oplus}\right) \\
51(34,78-\mathrm{HCN})\end{array}$ \\
\hline Cyclohexyl-(3-pyridyl)keton & $\begin{array}{l}3050 \mathrm{w}, 2940 \mathrm{~s} \\
2860 \mathrm{~s}, 1690 \mathrm{~s} \\
1585 \mathrm{~s}\end{array}$ & $\begin{array}{l}1.00-2.10(\mathrm{~m}, 10 \mathrm{H}) \\
3.20(\mathrm{~m}, 1 \mathrm{H}), 7.28(\mathrm{dd}, \\
\mathrm{H}-5), 8.07(\mathrm{dt}, \mathrm{H}-4), 8.60 \\
(\mathrm{dd}, \mathrm{H}-6), 9.03(\mathrm{~d}, \mathrm{H}-2)^{\mathrm{c})}\end{array}$ & $\begin{array}{l}25.41(2 \mathrm{C}), 25.60,28.90 \\
(2 \mathrm{C}), 45.74,123.37,131.25 \\
149.45,152.88,202.16(\mathrm{CO})\end{array}$ & $\begin{array}{l}189\left(35 \%, \mathrm{M}^{\oplus}\right), 157(17), \\
146\left(20, \mathrm{M}^{\oplus}-\mathrm{C}_{3} \mathrm{H}_{7}\right), 134 \\
\left(41, \mathrm{M}^{\oplus}-\mathrm{C}_{4} \mathrm{H}_{7}\right), 106 \\
\left(100, \mathrm{C}_{5} \mathrm{H}_{4} \mathrm{NCO}^{\oplus}\right), 83(19, \\
\left.\mathrm{C}_{6} \mathrm{H}_{11}^{\oplus}\right), 78\left(58, \mathrm{C}_{5} \mathrm{H}_{4} \mathrm{~N}^{\oplus}\right), \\
55\left(70, \mathrm{C}_{4} \mathrm{H}_{7}^{\oplus}\right)\end{array}$ \\
\hline
\end{tabular}


Tab. 5 (Fortsetzung)

\begin{tabular}{|c|c|c|c|c|}
\hline Keton & $\begin{array}{c}\mathrm{IR}^{\mathrm{a})} \\
{\left[\mathrm{cm}^{-1}\right]}\end{array}$ & $\begin{array}{c}{ }^{1} \mathrm{H}-\mathrm{NMR}\left(\mathrm{CDCl}_{3}\right)^{\mathrm{b})} \\
\delta \text {-Werte }\end{array}$ & $\begin{array}{c}{ }^{13} \mathrm{C} \text {-NMR }\left(\mathrm{CDCl}_{3}\right) \\
\delta \text {-Werte }\end{array}$ & $\begin{array}{c}\text { MS }(70 \mathrm{eV}) \\
m / e\end{array}$ \\
\hline $\begin{array}{l}\text { 3-(4-Trifluormethylbenzoyl)- } \\
\text { pyridin }\end{array}$ & $\begin{array}{l}3100 \mathrm{w}, 3060 \mathrm{w} \\
1665 \mathrm{~s}, 1590 \mathrm{~m}\end{array}$ & $\begin{array}{l}7.55(\mathrm{dd}, \mathrm{H}-5), 7.92(\mathrm{~m}, \\
\left.\mathrm{AA}^{\prime} \mathrm{BB}^{\prime}-\mathrm{Typ}, 4 \mathrm{H}\right), 8.20(\mathrm{dt} \\
\mathrm{H}-4), 8.92(\mathrm{dd}, \mathrm{H}-6), 9.06 \\
(\mathrm{~m}, \mathrm{H}-2)\end{array}$ & $\begin{array}{l}123.55,125.75\left(\mathrm{q}, J_{\mathrm{CF}}=\right. \\
3.6 \mathrm{~Hz}, 2 \mathrm{C}), 130.19(2 \mathrm{C}) \\
132.52,137.15,139.98, \\
151.03,153.47,193.77(\mathrm{CO})\end{array}$ & $\begin{array}{l}251\left(11 \%, \mathrm{M}^{\oplus}\right), 232(11, \\
\left.\mathrm{M}^{\oplus}-\mathrm{F}\right), 182\left(81, \mathrm{M}^{\oplus}-\right. \\
\left.\mathrm{CF}_{3}\right), 173\left(89, \mathrm{M}^{\oplus}-\right. \\
\left.\mathrm{C}_{5} \mathrm{H}_{4} \mathrm{~N}\right), 145(92,173- \\
\mathrm{CO}), 106\left(78, \mathrm{C}_{5} \mathrm{H}_{4} \mathrm{NCO}^{\oplus}\right), \\
78\left(100, \mathrm{C}_{5} \mathrm{H}_{4} \mathrm{~N}^{\oplus}\right), 51 \\
\left(92,78-\mathrm{HCN}^{2}\right)\end{array}$ \\
\hline $\begin{array}{l}\text { 3-(3-Trifluormethylbenzoyl)- } \\
\text { pyridin }\end{array}$ & $\begin{array}{l}3040 \mathrm{w}, 1665 \mathrm{~s} \\
1610 \mathrm{~m}, 1580 \mathrm{~m}\end{array}$ & $\begin{array}{l}7.49(\mathrm{dd}, \mathrm{H}-5), 7.67(\mathrm{t}, \\
\left.J=8 \mathrm{~Hz}, \mathrm{H}-5^{\prime}\right), 7.90(\mathrm{~d}, \\
\left.J=8 \mathrm{~Hz}, \mathrm{H}-4^{\prime}\right), 7.99(\mathrm{~d}, \\
\left.J=8 \mathrm{~Hz}, \mathrm{H}-6^{\prime}\right), 8.08(\mathrm{~s}, \\
\left.\mathrm{H}-2^{\prime}\right), 8.13(\mathrm{dt}, \mathrm{H}-4), 8.85 \\
\text { (dd, H-6), } 8.98(\mathrm{~m}, \mathrm{H}-2)\end{array}$ & $\begin{array}{l}123.57,126.62\left(\mathrm{q}, J_{\mathrm{CF}}=\right. \\
3.8 \mathrm{~Hz}), 129.32,129.53(\mathrm{q} \\
\left.J_{\mathrm{CF}}=3.7 \mathrm{~Hz}\right), 132.46, \\
133.08,137.09,137.51 \\
150.89,153.42,193.41(\mathrm{CO})\end{array}$ & $\begin{array}{l}251\left(100 \%, \mathrm{M}^{\oplus}\right), 231(18, \\
\left.\mathrm{M}^{\oplus}-\mathrm{HF}\right), 182\left(62, \mathrm{M}^{\oplus}-\right. \\
\left.\mathrm{CF}_{3}\right), 173\left(81, \mathrm{M}^{\oplus}-\right. \\
\left.\mathrm{C}_{5} \mathrm{H}_{4} \mathrm{~N}^{\oplus}\right), 145(63,173- \\
\mathrm{CO}), 106\left(30, \mathrm{C}_{5} \mathrm{H}_{4} \mathrm{NCO}^{\oplus}\right), \\
78\left(41, \mathrm{C}_{5} \mathrm{H}_{4} \mathrm{~N}^{\oplus}\right), 51(33, \\
78-\mathrm{HCN})\end{array}$ \\
\hline 4-Oxo-4-(3-pyridyl)butannitril & $\begin{array}{l}3050 \mathrm{w}, 2980 \mathrm{w}, \\
2930 \mathrm{w}, 2240 \mathrm{~m}, \\
1685 \mathrm{~s}, 1585 \mathrm{~s}\end{array}$ & $\begin{array}{l}2.79(\mathrm{t}, J=7 \mathrm{~Hz}, 2 \mathrm{H}) \\
3.47(\mathrm{t}, J=7 \mathrm{~Hz}, 2 \mathrm{H}), 7.47 \\
(\mathrm{dd}, \mathrm{H}-5), 8.28(\mathrm{dt}, \mathrm{H}-4), \\
8.73(\mathrm{~m}, \mathrm{H}-6), 9.11(\mathrm{~m}, \mathrm{H}-2)\end{array}$ & $\begin{array}{l}11.35\left(-C \mathrm{H}_{2}-\mathrm{CN}\right), 34.30 \\
\left(-\mathrm{CO}-C \mathrm{H}_{2}-\right), 118.81 \\
(\mathrm{CN}), 123.62,130.85 \\
135.11,149.27,153.96 \\
194.43(\mathrm{CO})\end{array}$ & $\begin{array}{l}160\left(10 \%, \mathrm{M}^{\oplus}\right), 107(100 \\
\left.\mathrm{M}^{\oplus}-\mathrm{CH}_{2}=\mathrm{CHCN}\right), 78 \\
\left(80, \mathrm{C}_{5} \mathrm{H}_{4} \mathrm{~N}^{\oplus}\right), 51(52 \\
78-\mathrm{HCN})\end{array}$ \\
\hline
\end{tabular}

a) Flüssigkeiten als Filme, Feststoffe in $\mathrm{KBr}$; angegeben sind die charakteristischen Banden. - b) Alle Spektren werden nach 1. Ordnung interpretiert; ungefähre Kopplungskonstanten zwischen Pyridinprotonen: $J_{54}=8 \mathrm{~Hz}, J_{65}=5 \mathrm{~Hz}, J_{64}=J_{42}=2 \mathrm{~Hz}, J_{52}=1 \mathrm{~Hz}$ (nur bei wenigen Verbindungen aufgelöst). - c) In $\mathrm{CCl}_{4}$. 


\section{3,3-Dimethyl-1-(3-pyridyl)-1-butanon}

1) 3,3-Dimethyl-1-(3-pyridyl)-1-butanol: Aus $75.5 \mathrm{~g}$ (0.50 mol) 1-Brom-2,2-dimethylpropan ${ }^{31,32)}$ und $12.3 \mathrm{~g}(0.50 \mathrm{~mol})$ Magnesium in $400 \mathrm{ml}$ absol. Ether wird das Grignard-Reagens hergestellt. Unter Schutzgas $\left(\mathrm{N}_{2}\right)$ und Eiskühlung versetzt man die gerührte Grignard-Lösung tropfenweise mit $28.2 \mathrm{~g}(0.45 \mathrm{~mol}) 3$-Pyridincarbaldehyd in $300 \mathrm{ml}$ absol. Ether. Zur Vervollständigung der Umsetzung rührt man noch ca. $12 \mathrm{~h}$. - Man gießt auf eine Mischung aus etwa $100 \mathrm{~g}$ Ammoniumchlorid und $400 \mathrm{~g}$ Eis/Wasser, trennt die etherische Phase ab und extrahiert die wäßrige noch zweimal mit je $150 \mathrm{ml}$ Ether. Die vereinigten Etherextrakte werden mit 50-100 ml Wasser gewaschen, mit Magnesiumsulfat getrocknet und fraktioniert. Ausb. $45 \%$, Sdp. $158^{\circ} \mathrm{C} /$ 10 Torr, $n_{\mathrm{D}}^{20}=1.5065$, Pikrat (gelbe Nadeln aus Ethanol/Wasser); Schmp. $130^{\circ} \mathrm{C}$. - IR (Film): 3200 (s, breit, OH), 2950, 2905, 2860 (s, aliphat. C - H), 1590, $1580\left(\mathrm{~m}\right.$, aromat. C - C) $\mathrm{cm}^{-1}$. ${ }^{1} \mathrm{H}-\mathrm{NMR}\left(\mathrm{CDCl}_{3}\right): \delta=0.98(\mathrm{~s}, 9 \mathrm{H}), 1.54\left(\mathrm{dd}, J_{1}=15 \mathrm{~Hz}, J_{2}=4 \mathrm{~Hz}, 1 \mathrm{H}\right), 1.78\left(\mathrm{dd}, J_{1}=\right.$ $\left.15 \mathrm{~Hz}, J_{3}=9 \mathrm{~Hz}, 1 \mathrm{H}\right), 3.41(\mathrm{~s}$, breit, $\mathrm{OH}), 4.83\left(\mathrm{dd}, J_{2}=4 \mathrm{~Hz}, J_{3}=9 \mathrm{~Hz}, 1 \mathrm{H}\right), 7.23$ (dd, $J_{54}=$ $\left.8 \mathrm{~Hz}, J_{65}=4 \mathrm{~Hz}, \mathrm{H}-5\right), 7.71\left(\mathrm{dt}, J_{54}=8 \mathrm{~Hz}, J_{64}=J_{42}=2 \mathrm{~Hz}, \mathrm{H}-4\right), 8.36$ (m, breit, H-6), 8.41 (m, breit, H-2). $-{ }^{13} \mathrm{C}-\mathrm{NMR}\left(\mathrm{CDCl}_{3}\right): \delta=30.27\left[\mathrm{C}\left(\mathrm{CH}_{3}\right)_{3}\right], 30.60\left[\mathrm{C}\left(\mathrm{CH}_{3}\right)_{3}, 52.81\left(-\mathrm{CH}_{2}-\right)\right.$, $69.51(-\mathrm{CH}(\mathrm{OH})-$ ] $, 123.54,133.94,142.67,147.48,147.89$ (aromat. C). - Pikrat:

$$
\mathrm{C}_{17} \mathrm{H}_{20} \mathrm{~N}_{4} \mathrm{O}_{8} \text { (408.4) Ber. C } 50.00 \mathrm{H} 4.94 \text { N } 13.72 \text { Gef. C } 49.93 \mathrm{H} 4.96 \text { N } 13.55
$$

2) 3,3-Dimethyl-1-(3-pyridyl)-1-butanon: In Anlehnung an Lit. ${ }^{25)}$ werden $4.1 \mathrm{~g}(27 \mathrm{mmol})$ 3,3-Dimethyl-1-(3-pyridyl)-1-butanol in $25 \mathrm{ml}$ absol. Tetrachlorkohlenstoff mit $4.8 \mathrm{~g}$ (27 mmol) $\mathrm{N}$-Bromsuccinimid sowie $0.5 \mathrm{~g}$ Calciumcarbonat versetzt und $4 \mathrm{~h}$ lang auf $65^{\circ} \mathrm{C}$ erhitzt. Nach Stehenlassen (ca. $12 \mathrm{~h}$ ) fügt man ca. $50 \mathrm{ml} 2 \mathrm{~N} \mathrm{NaOH}$ und ca. $50 \mathrm{ml}$ Chloroform hinzu, wäscht die vereinigten organischen Extrakte mit $50 \mathrm{ml}$ Wasser und trocknet sie mit Magnesiumsulfat. Das Lösungsmittel wird abdestilliert und der Rückstand bei 0.05 Torr fraktioniert. Ausb. 55\%, physikalische Daten siehe Tab. 4; spektroskopische Daten siehe Tab. 5.

5-Acyl-2-pyridincarboxamide $4\left(R^{I}=R^{2}=H\right): 0.1 \mathrm{~mol} 3$-Acylpyridin 3 in $70 \mathrm{ml}$ Formamid werden bei $0-5^{\circ} \mathrm{C}$ unter Rühren mit $9.6 \mathrm{ml}(0.17 \mathrm{~mol})$ konz. $\mathrm{H}_{2} \mathrm{SO}_{4}$ versetzt. Unter Schutzgas fügt man $56.0 \mathrm{~g}(0.20 \mathrm{~mol})$ Eisen(II)-sulfat-heptahydrat hinzu und tropft innerhalb von $1-2 \mathrm{~h}$ $22.5 \mathrm{~g}(0.20 \mathrm{~mol})$ 80proz. tert-Butylhydroperoxid (Fa. Peroxid-Chemie, München-Höllriegelskreuth) ein, wobei die Temp. durch Kühlung mit einem Eisbad unterhalb von $10^{\circ} \mathrm{C}$ gehalten wird. Im Anschluß daran rührt man noch $1 \mathrm{~h}$ bei Raumtemp. Man versetzt mit einer Kaliumcitratlösung aus $56.1 \mathrm{~g}(1.00 \mathrm{~mol})$ Kaliumhydroxid, $69.1 \mathrm{~g}(0.36 \mathrm{~mol})$ Citronensäure und $400 \mathrm{ml}$ Wasser. Gegebenenfalls fügt man noch verdünnte Kaliumhydroxidlösung oder Kaliumcarbonatlösung unter starkem Rühren zu, bis die Reaktionsmischung deutlich alkalisch ist und verdünnt mit Wasser, um ausgefallene Salze zu lösen. Das Reaktionsprodukt fällt häufig in flockiger Form an, wird dann abgesaugt, mit Wasser gewaschen und zur Entfernung von Wasser und Spuren an tert-Butylhydroperoxid über Phosphorpentoxid getrocknet. Andernfalls wird mit Chloroform extrahiert $(3 \times 200 \mathrm{ml})$, dem zwecks einer besseren Phasentrennung und einer besseren Löslichkeit des Produktes 20-30\% Isopropylalkohol zugesetzt werden können. Die vereinigten organischen Extrakte wäscht man mit ca. $100 \mathrm{ml}$ Wasser und trocknet sie mit 3-Å-Molekularsieb oder Natriumsulfat (Magnesiumsulfat eignet sich weniger, da es einen Teil des Produktes zurückhält) und entfernt das Lösungsmittel i. Vak. Das zurückbleibende Rohprodukt wird, wie oben erwähnt, über Phosphorpentoxid getrocknet. Anschließend wird aus Ethanol oder Methanol umkristallisiert. Ausb., physikalische und spektroskopische Daten siehe Tab. 6 und 7.

5-Butanoyl-N,N-dimethyl-2-pyridincarboxamid und 5-Butanoyl-N-tert-butyl-2-pyridincarboxamid: 1-(3-Pyridyl)-1-butanon wird völlig analog zur oben beschriebenen Synthesevorschrift für 5-Acyl-2-pyridincarboxamide $4\left(\mathrm{R}^{1}=\mathrm{R}^{2}=\mathrm{H}\right)$ mit DMF bzw. $N$-tert-Butylformamid (aus Ameisensäure-ethylester und tert-Butylamin nach Lit. ${ }^{33)}$ ) umgesetzt. Zur Isolierung der Reak- 
tionsprodukte wird zunächst DMF bzw. N-tert-Butylformamid sowie unumgesetztes 1-(3-Pyridyl)1-butanon bei 15 Torr abdestilliert. 5-Butanoyl- $N, N$-dimethyl-2-pyridincarboxamid erhält man durch Filtration des teerigen Rückstandes in Essigsäure-ethylester über neutrales Aluminiumoxid (Aktivitätsstufe I). Ausb., physikalische und spektroskopische Daten siehe Tab. 6 und 7.

Tab. 6. Ausbeuten und analytische Daten der 5-Acyl-2-pyridincarboxamide 4

\begin{tabular}{|c|c|c|c|c|c|c|c|}
\hline \multirow{2}{*}{$\begin{array}{l}\ldots \text {-2-pyridin- } \\
\text { carboxamid }\end{array}$} & \multirow{2}{*}{ Ausb. a) } & \multirow{2}{*}{ Schmp. b) } & \multirow{2}{*}{$\begin{array}{l}\text { Summenformel } \\
\text { (Molmasse) }\end{array}$} & & \multicolumn{3}{|c|}{ Analyse } \\
\hline & & & & & C & $\mathrm{H}$ & $\mathrm{N}$ \\
\hline 5-Benzoyl-... & 45 & $147-155$ & $\begin{array}{l}\mathrm{C}_{13} \mathrm{H}_{10} \mathrm{~N}_{2} \mathrm{O}_{2} \\
\quad(226.2)\end{array}$ & $\begin{array}{l}\text { Ber. } \\
\text { Gef. }\end{array}$ & $\begin{array}{l}69.02 \\
69.08\end{array}$ & $\begin{array}{l}4.46 \\
4.54\end{array}$ & $\begin{array}{l}12.38 \\
12.52\end{array}$ \\
\hline 5-(4-Chlorbenzoyl)-... & 80 & 203 & $\begin{array}{l}\mathrm{C}_{13} \mathrm{H}_{9} \mathrm{ClN}_{2} \mathrm{O}_{2} \\
(260.7)\end{array}$ & $\begin{array}{l}\text { Ber. } \\
\text { Gef. }\end{array}$ & $\begin{array}{l}59.90 \\
59.96\end{array}$ & $\begin{array}{l}3.48 \\
3.47\end{array}$ & $\begin{array}{l}10.75 \\
10.73\end{array}$ \\
\hline 5-(4-Fluorbenzoyl)-... & 79 & 178 & $\begin{array}{l}\mathrm{C}_{13} \mathrm{H}_{9} \mathrm{FN}_{2} \mathrm{O}_{2} \\
(244.2)\end{array}$ & $\begin{array}{l}\text { Ber. } \\
\text { Gef. }\end{array}$ & $\begin{array}{l}63.93 \\
64.02\end{array}$ & $\begin{array}{l}3.71 \\
3.81\end{array}$ & $\begin{array}{l}11.47 \\
11.30\end{array}$ \\
\hline 5-(4-Methoxybenzoyl)-. & 57 & 217 & $\begin{array}{l}\mathrm{C}_{14} \mathrm{H}_{12} \mathrm{~N}_{2} \mathrm{O}_{3} \\
(256.3)\end{array}$ & $\begin{array}{l}\text { Ber.c) } \\
\text { Gef.c) }\end{array}$ & $\begin{array}{l}65.62 \\
64.93\end{array}$ & $\begin{array}{l}4.72 \\
4.90\end{array}$ & $\begin{array}{l}10.93 \\
10.75\end{array}$ \\
\hline 5-(4-tert-Butylbenzoyl)-. & 82 & 201 & $\begin{array}{c}\mathrm{C}_{17} \mathrm{H}_{18} \mathrm{~N}_{2} \mathrm{O}_{2} \\
(282.3)\end{array}$ & $\begin{array}{l}\text { Ber. } \\
\text { Gef. }\end{array}$ & $\begin{array}{l}72.32 \\
72.12\end{array}$ & $\begin{array}{l}6.43 \\
6.32\end{array}$ & $\begin{array}{l}9.92 \\
9.86\end{array}$ \\
\hline 5-Butanoyl-... & 31 & 148 & $\begin{array}{c}\mathrm{C}_{10} \mathrm{H}_{12} \mathrm{~N}_{2} \mathrm{O}_{2} \\
(192.2)\end{array}$ & $\begin{array}{l}\text { Ber. } \\
\text { Gef. }\end{array}$ & $\begin{array}{l}62.49 \\
62.61\end{array}$ & $\begin{array}{l}6.29 \\
6.31\end{array}$ & $\begin{array}{l}14.57 \\
14.62\end{array}$ \\
\hline 5-Isovaleroyl-... d) & 20 & 143 & $\begin{array}{c}\mathrm{C}_{11} \mathrm{H}_{14} \mathrm{~N}_{2} \mathrm{O}_{2} \\
\quad(206.2)\end{array}$ & $\begin{array}{l}\text { Ber. } \\
\text { Gef. }\end{array}$ & $\begin{array}{l}64.06 \\
66.29\end{array}$ & $\begin{array}{l}6.84 \\
6.77\end{array}$ & $\begin{array}{l}13.58 \\
13.47\end{array}$ \\
\hline $\begin{array}{l}\text { 5-(3,3-Dimethyl- } \\
\text { butanoyl)-... }\end{array}$ & 27 & 178 & $\begin{array}{l}\mathrm{C}_{12} \mathrm{H}_{16} \mathrm{~N}_{2} \mathrm{O}_{2} \\
\quad(220.3)\end{array}$ & $\begin{array}{l}\text { Ber. } \\
\text { Gef. }\end{array}$ & $\begin{array}{l}65.39 \\
65.16\end{array}$ & $\begin{array}{l}7.32 \\
7.39\end{array}$ & $\begin{array}{l}12.72 \\
12.74\end{array}$ \\
\hline $\begin{array}{l}\text { 5-(4-Trifluormethyl- } \\
\text { benzoyl)-... }\end{array}$ & 52 & $\begin{array}{l}200 \\
\text { (Zers.) }\end{array}$ & $\begin{array}{l}\mathrm{C}_{14} \mathrm{H}_{9} \mathrm{~F}_{3} \mathrm{~N}_{2} \mathrm{O}_{2} \\
\quad(294.2)\end{array}$ & $\begin{array}{l}\text { Ber. } \\
\text { Gef. }\end{array}$ & $\begin{array}{l}57.15 \\
57.20\end{array}$ & $\begin{array}{l}3.08 \\
3.00\end{array}$ & $\begin{array}{l}9.52 \\
9.59\end{array}$ \\
\hline $\begin{array}{l}\text { 5-(3-Trifluormethyl- } \\
\text { benzoyl)-... }\end{array}$ & 50 & $\begin{array}{l}228 \\
\text { (Zers.) }\end{array}$ & $\begin{array}{l}\mathrm{C}_{14} \mathrm{~N}_{9} \mathrm{~F}_{3} \mathrm{~N}_{2} \mathrm{O}_{2} \\
\quad(294.2)\end{array}$ & $\begin{array}{l}\text { Ber. } \\
\text { Gef. }\end{array}$ & $\begin{array}{l}57.15 \\
57.13\end{array}$ & $\begin{array}{l}3.08 \\
3.07\end{array}$ & $\begin{array}{l}9.52 \\
9.56\end{array}$ \\
\hline 5-(3-Cyanpropanoyl)-... & 91 & $\begin{array}{l}210 \\
\text { (Zers.) }\end{array}$ & $\begin{array}{l}\mathrm{C}_{10} \mathrm{H}_{9} \mathrm{~N}_{3} \mathrm{O}_{2} \\
(203.2)\end{array}$ & $\begin{array}{l}\text { Ber. } \\
\text { Gef. }\end{array}$ & $\begin{array}{l}59.11 \\
58.93\end{array}$ & $\begin{array}{l}4.46 \\
4.42\end{array}$ & $\begin{array}{l}20.68 \\
20.69\end{array}$ \\
\hline 5-(3-Nitrobenzoyl)-... & 46 & $\begin{array}{l}195-198 \\
\text { (Zers.) }\end{array}$ & $\begin{array}{l}\mathrm{C}_{13} \mathrm{H}_{9} \mathrm{~N}_{3} \mathrm{O}_{4} \\
(271.2)\end{array}$ & $\begin{array}{l}\text { Ber. } \\
\text { Gef. }\end{array}$ & $\begin{array}{l}57.57 \\
57.47\end{array}$ & $\begin{array}{l}3.34 \\
3.32\end{array}$ & $\begin{array}{l}15.49 \\
15.43\end{array}$ \\
\hline $\begin{array}{l}\text { 5-Butanoyl- } N, N \text {-di- } \\
\text { methyl-... }\end{array}$ & 20 & 92 & $\begin{array}{l}\mathrm{C}_{12} \mathrm{H}_{16} \mathrm{~N}_{2} \mathrm{O}_{2} \\
\quad(220.3)\end{array}$ & $\begin{array}{l}\text { Ber. } \\
\text { Gef. }\end{array}$ & $\begin{array}{l}65.43 \\
65.46\end{array}$ & $\begin{array}{l}7.32 \\
7.35\end{array}$ & $\begin{array}{l}12.72 \\
12.62\end{array}$ \\
\hline $\begin{array}{l}\text { 5-Butanoyl-N-tert- } \\
\text { butyl-... }\end{array}$ & 2 & 68 & $\begin{array}{l}\mathrm{C}_{14} \mathrm{H}_{20} \mathrm{~N}_{2} \mathrm{O}_{2} \\
(248.3)\end{array}$ & $\begin{array}{l}\text { Ber. } \\
\text { Gef. }\end{array}$ & $\begin{array}{l}67.72 \\
67.60\end{array}$ & $\begin{array}{l}8.12 \\
8.14\end{array}$ & $\begin{array}{l}11.28 \\
11.25\end{array}$ \\
\hline
\end{tabular}

a) Ausb, in \% nach Umkristallisation. - b) In ${ }^{\circ} \mathrm{C}$, alle Substanzen sind aus Ethanol umkristallisiert. - c) Ber. O 18.73, Gef. O 18.53. - d) Isoliert durch Chromatographie mit Methylenchlorid/Acetonitril (3:1) an Kieselgel als zweite Fraktion nach 5-Isovaleroyl-2,4-pyridindicarboxamid.

Bei der Reaktion mit tert-Butylformamid werden entsprechend durch Chromatographie an Kieselgel (Essigester: Pentan $=1: 5$ ) nacheinander die folgenden Produkte erhalten:

3-Butanoyl-N-tert-butyl-2-pyridincarboxamid: Ausb. ca. 1\%; IR $\left(\mathrm{CDCl}_{3}\right): 3430(\mathrm{~m}, \mathrm{~N}-\mathrm{H}$ intra), $3380\left(\mathrm{~s}, \mathrm{~N}-\mathrm{H}\right.$ inter). $-{ }^{1} \mathrm{H}-\mathrm{NMR}\left(\mathrm{CDCl}_{3}\right): \delta=7.45\left(\mathrm{dd}, J_{54}=8 \mathrm{~Hz}, J_{65}=4.5 \mathrm{~Hz}, \mathrm{H}-5\right), 7.60$ (dd, $\left.J_{54}=8 \mathrm{~Hz}, J_{64}=2 \mathrm{~Hz}, \mathrm{H}-4\right), 8.56\left(\mathrm{dd}, J_{65}=4.5 \mathrm{~Hz}, J_{64}=2 \mathrm{~Hz}, \mathrm{H}-6\right) .-$ MS $(70 \mathrm{eV})$ : $m / e=248\left(17 \%, \mathrm{M}^{\oplus}\right), 205\left(100 \%, \mathrm{M}^{\oplus}-\mathrm{C}_{3} \mathrm{H}_{7}\right), 149\left(70 \%, \mathrm{M}^{\oplus}-\mathrm{C}_{4} \mathrm{H}_{9} \mathrm{NCO}\right)$.

5-Butanoyl-N-tert-butyl-2-pyridincarboxamid: (Ausb., physikalische und spektroskopische Daten siehe Tab. 6 und 7).

3-Butanoyl-N-tert-butyl-4-pyridincarboxamid: Ausb. ca. 3\%; IR (KBr): 3450 (s, N-H), 2970, 2930 (w, C-H), 1690, 1645 (s, Schulter, C = O und Amid I), 1585, 1560 (m, Amid II und C - C).

Liebigs Ann. Chem. 1982 
Tab. 7. Spektroskopische Daten der 5-Acyl-2-pyridincarboxamide 4

\begin{tabular}{|c|c|c|c|c|}
\hline$\ldots$-2-pyridincarboxamid & $\begin{array}{l}\text { IR }(\mathrm{KBr})^{\mathrm{a})} \\
{\left[\mathrm{cm}^{-1}\right]}\end{array}$ & $\begin{array}{c}{ }^{1} \mathrm{H}-\mathrm{NMR}\left(\mathrm{CDCl}_{3}\right)^{\mathrm{b})} \\
\delta \text {-Werte }\end{array}$ & $\begin{array}{c}{ }^{13} \mathrm{C}-\mathrm{NMR}\left(\mathrm{CDCl}_{3}\right) \\
\delta \text {-Werte }\end{array}$ & $\begin{array}{c}\mathrm{MS}(70 \mathrm{eV}) \\
m / e\end{array}$ \\
\hline 5-Benzoyl-... & $\begin{array}{l}3460 \mathrm{~m}, 3430 \mathrm{~m} \\
3060 \mathrm{w}, 1690 \mathrm{~s} \\
1650 \mathrm{~s}, 1590 \mathrm{~s}\end{array}$ & $\begin{array}{l}7.52-7.87(\mathrm{~m}, 5 \mathrm{H}), 8.27 \\
(\mathrm{dd}, \mathrm{H}-4), 8.33(\mathrm{dd}, \mathrm{H}-3) \\
8.97(\mathrm{~m}, \mathrm{H}-6)^{\mathrm{c})}\end{array}$ & $\begin{array}{l}122.25,129.04(2 \mathrm{C}), \\
130.28(2 \mathrm{C}), 133.91,135.67 \\
136.58,139.91,149.73, \\
152.15,194.86(\mathrm{CO})^{\mathrm{c})}\end{array}$ & $\begin{array}{l}226\left(41 \%, \mathrm{M}^{\oplus}\right), 209(20, \\
\left.\mathrm{M}^{\oplus}-\mathrm{NH}_{3}\right), 183(57, \\
\mathrm{M}^{\oplus}-\mathrm{HNCO}^{\oplus}, 149(9, \\
\left.\mathrm{M}^{\oplus}-\mathrm{C}_{6} \mathrm{H}_{5}\right), 105(100, \\
\left.\mathrm{C}_{6} \mathrm{H}_{5} \mathrm{CO}^{\oplus}\right), 77\left(89, \mathrm{C}_{6} \mathrm{H}_{5}^{\oplus}\right), \\
44\left(15, \mathrm{H}_{2} \mathrm{NCO}^{\oplus}\right)\end{array}$ \\
\hline 5-(4-Chlorbenzoyl)-... & $\begin{array}{l}3450 \mathrm{~m}, 1690 \mathrm{~s} \\
1650 \mathrm{~s}, 1590 \mathrm{~s}\end{array}$ & $\begin{array}{l}\left.7.59 \text { (mc, } \mathrm{AA}^{\prime} \mathrm{BB}^{\prime}-\mathrm{Typ}, 4 \mathrm{H}\right) \\
8.17(\mathrm{dd}, \mathrm{H}-4), 8.25(\mathrm{dd} \\
\mathrm{H}-3), 8.86(\mathrm{~m}, \mathrm{H}-6)^{\mathrm{c})}\end{array}$ & - & $\begin{array}{l}260\left(24 \%, \mathrm{M}^{\oplus}\right), 243(2, \\
\left.\mathrm{M}^{\oplus}-\mathrm{NH}_{3}\right), 225(100, \\
\left.\mathrm{M}^{\oplus}-\mathrm{Cl}\right), 217(44, \\
\left.\mathrm{M}^{\oplus}-\mathrm{HNCO}\right), 139(60, \\
\left.\left.\mathrm{Cl}-\mathrm{C}_{6} \mathrm{H}_{4} \mathrm{CO}\right)^{\oplus}\right), 111(30, \\
139-\mathrm{CO}), 44(9, \\
\left.\mathrm{H}_{2} \mathrm{NCO}^{\oplus}\right)\end{array}$ \\
\hline 5-(4-Fluorbenzoyl)-... & $\begin{array}{l}3450 \mathrm{~m}, 3420 \mathrm{~m} \\
1690 \mathrm{~s}, 1645 \mathrm{~s} \\
1605 \mathrm{~m}\end{array}$ & $\begin{array}{l}7.21(\mathrm{mc}, 2 \mathrm{H}), 7.84(\mathrm{mc}, \\
2 \mathrm{H}), 8.81(\mathrm{dd}, \mathrm{H}-4), 8.24 \\
\left.(\mathrm{dd}, \mathrm{H}-3), 8.87(\mathrm{~m}, \mathrm{H}-6)^{\mathrm{d}}\right)\end{array}$ & $\begin{array}{l}115.85\left(\mathrm{~d}, J_{\mathrm{CF}}=22 \mathrm{~Hz}\right. \\
2 \mathrm{C}), 121.64,132.68(\mathrm{~d} \\
\left.J_{\mathrm{CF}}=10 \mathrm{~Hz}, 2 \mathrm{C}\right), 132.69 \\
134.74,138.21,148.82 \\
152.63,165.28,165.45 \\
\left(\mathrm{~d}, J_{\mathrm{CF}}=254 \mathrm{~Hz}\right), 198.32 \\
\left.(\mathrm{CO})^{\mathrm{d}}\right)\end{array}$ & $\begin{array}{l}244\left(54 \%, \mathrm{M}^{\oplus}\right), 227(77, \\
\left.\mathrm{M}^{\oplus}-\mathrm{NH}_{3}\right), 201(90, \\
\mathrm{M}^{\oplus}-\mathrm{HNCO}^{\oplus}, 149(11, \\
\left.\mathrm{M}^{\oplus}-\mathrm{FC}_{6} \mathrm{H}_{4}\right), 123(100, \\
\left.\mathrm{FC}_{6} \mathrm{H}_{4} \mathrm{CO}^{\oplus}\right), 95(60, \\
\left.\mathrm{FC}_{6} \mathrm{H}_{4}^{\oplus}\right), 78\left(12, \mathrm{C}_{5} \mathrm{H}_{4} \mathrm{~N}^{\oplus}\right) \\
51(11,78-\mathrm{HCN}), 44(14, \\
\left.\mathrm{H}_{2} \mathrm{NCO}^{\oplus}\right)\end{array}$ \\
\hline 5-(4-Methoxybenzoyl)-... & $\begin{array}{l}3430 \mathrm{~s}, 1700 \mathrm{~s} \\
1640 \mathrm{~s}, 1590 \mathrm{~s}\end{array}$ & $\begin{array}{l}3.94\left(\mathrm{~s}, \mathrm{OCH}_{3}\right), 7.54(\mathrm{mc}, \\
\left.\mathrm{AA}^{\prime} \mathrm{BB}^{\prime}-\mathrm{Typ}, 4 \mathrm{H}\right), 7.83 \\
\text { und } 8.03\left(\mathrm{~s}, \text { breit, } \mathrm{NH}_{2}\right) \\
8.27(\mathrm{dd}, \mathrm{H}-3), 8.33(\mathrm{dd}, \\
\left.\mathrm{H}-4), 8.92(\mathrm{~m}, \mathrm{H}-6)^{\mathrm{e}}\right)\end{array}$ & - & $\begin{array}{l}256\left(37 \%, \mathrm{M}^{\oplus}\right), 225(8, \\
\left.\mathrm{M}^{\oplus}-\mathrm{OCH}_{3}\right), 213(11, \\
\mathrm{M}^{\oplus}-\mathrm{HNCO}^{\oplus}, 135(100, \\
\left.\mathrm{CH}_{3} \mathrm{OC}_{6} \mathrm{H}_{4} \mathrm{CO}^{\oplus}\right), 107(16 \\
135-\mathrm{CO}), 44(8, \\
\left.\mathrm{H}_{2} \mathrm{NCO}^{\oplus}\right)\end{array}$ \\
\hline 5-(4-tert-Butylbenzoyl)-... & $\begin{array}{l}3410 \mathrm{~m}, 3240 \mathrm{~m}, \\
2970 \mathrm{w}, 1650 \mathrm{~s} \\
\text { (Schulter), } \\
1605 \mathrm{~m}\end{array}$ & 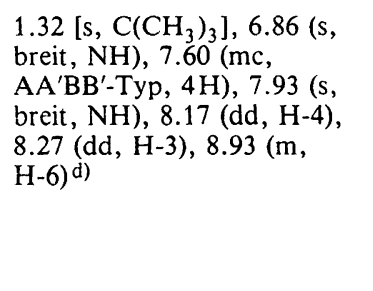 & $\begin{array}{l}31.13(3 \mathrm{C}), 121.89,125.69 \\
125.94(2 \mathrm{C}), 130.25(2 \mathrm{C}) \\
134.15,138.57,149.41 \\
152.73,157.40,165.76 \\
\left(\mathrm{CONH}_{2}\right), 206.38(\mathrm{CO})\end{array}$ & $\begin{array}{l}282\left(33 \%, \mathrm{M}^{\oplus}\right), 267(100, \\
\left.\mathrm{M}^{\oplus}-\mathrm{CH}_{3}\right), 239(6, \\
\mathrm{M}^{\oplus}-\mathrm{HNCO}^{\oplus}, 226(7, \\
\left.\mathrm{M}^{\oplus}-\mathrm{C}_{4} \mathrm{H}_{8}\right), 161[12, \\
\left.\left(\mathrm{CH}_{3}\right)_{3} \mathrm{C}-\mathrm{C}_{6} \mathrm{H}_{4} \mathrm{CO}^{\oplus}\right], 149 \\
(22,161-\mathrm{CO}), 121(11, \\
\left.\mathrm{M}^{\oplus}-161\right), 78(13, \\
\left.\mathrm{C}_{5} \mathrm{H}_{4} \mathrm{~N}^{\oplus}\right), 44(14, \\
\left.\mathrm{H}_{2} \mathrm{NCO}^{\oplus}\right)\end{array}$ \\
\hline
\end{tabular}




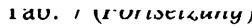

\begin{tabular}{|c|c|c|c|c|}
\hline$\ldots$-2-pyridincarboxamid & $\begin{array}{c}\text { IR }(\mathrm{KBr})^{a)} \\
{\left[\mathrm{cm}^{-1}\right]}\end{array}$ & $\begin{array}{c}{ }^{1} \mathrm{H}-\mathrm{NMR}\left(\mathrm{CDCl}_{3}\right)^{\mathrm{b})} \\
\delta \text {-Werte }\end{array}$ & $\begin{array}{c}{ }^{13} \mathrm{C} \text {-NMR }\left(\mathrm{CDCl}_{3}\right) \\
\delta \text {-Werte }\end{array}$ & $\begin{array}{c}\mathrm{MS}(70 \mathrm{eV}) \\
m / e\end{array}$ \\
\hline 5-Butanoyl-... & $\begin{array}{l}3400 \mathrm{~m}, 3220 \mathrm{~m}, \\
2970 \mathrm{w}, 2940 \mathrm{w} \\
2880 \mathrm{w}, 1680 \mathrm{~s} \\
1590 \mathrm{~m}, 1560 \mathrm{~m}\end{array}$ & $\begin{array}{l}1.05\left(\mathrm{t}, J=7 \mathrm{~Hz},-\mathrm{CH}_{3}\right), \\
1.82(\mathrm{sext}, J=7 \mathrm{~Hz}, \\
\left.-\mathrm{CH}-\mathrm{CH}_{2}\right), 3.05(\mathrm{t}, J= \\
\left.7 \mathrm{~Hz}, \mathrm{CO}-\mathrm{CH}_{2}-\right), 7.06 \\
\text { und } 8.10\left(\mathrm{~s}, \text { breit, } \mathrm{NH}_{2}\right) \text {, } \\
8.27(\mathrm{dd}, \mathrm{H}-3), 8.42(\mathrm{dd}, \\
\mathrm{H}-4), 9.15(\mathrm{~m}, \mathrm{H}-6)^{\mathrm{c})}\end{array}$ & $\begin{array}{l}13.75\left(-\mathrm{CH}_{3}\right), 17.41 \\
\left(\mathrm{CH}_{2}-\mathrm{CH}_{3}\right), 41.12 \\
\left(\mathrm{CO}-\mathrm{CH}_{2}-\right), 122.42 \\
134.21,136.83,148.29 \\
152.30,165.75\left(\mathrm{CONH}_{2}\right) \\
198.75(\mathrm{CO})\end{array}$ & $\begin{array}{l}192\left(15 \%, \mathrm{M}^{\oplus}\right), 175(20, \\
\left.\mathrm{M}^{\oplus}-\mathrm{NH}_{3}\right), 164(24, \\
\left.\mathrm{M}^{\oplus}-\mathrm{C}_{2} \mathrm{H}_{4}\right), 149(100, \\
\left.\mathrm{M}^{\oplus}-\mathrm{C}_{3} \mathrm{H}_{7} \mathrm{CO}\right), 121(29,78(19, \\
\left.\mathrm{C}_{5} \mathrm{H}_{4} \mathrm{~N}^{\oplus}\right), 44(15, \\
\left.\mathrm{H}_{2} \mathrm{NCO}^{\oplus}\right)\end{array}$ \\
\hline 5-Isovaleroyl-... & $\begin{array}{l}3410 \mathrm{~m}, 2955 \mathrm{w} \\
1675 \mathrm{~s}, 1590 \mathrm{~m}\end{array}$ & $\begin{array}{l}1.02(\mathrm{~d}, J=8 \mathrm{~Hz}, 6 \mathrm{H}), \\
2.31(\mathrm{mc}, J=8 \mathrm{~Hz}, 1 \mathrm{H}) \\
2.89(\mathrm{~d}, J=8 \mathrm{~Hz}, 2 \mathrm{H}) \\
6.03 \text { und } 7.89(\mathrm{~s}, \text { breit } \\
\left.\mathrm{NH}_{2}\right), 8.30(\mathrm{dd}, \mathrm{H}-3), 8.38 \\
(\mathrm{dd}, \mathrm{H}-4), 9.10(\mathrm{~m}, \mathrm{H}-6)\end{array}$ & - & $\begin{array}{l}206\left(8 \%, M^{\oplus}\right), 191(8, \\
\left.M^{\oplus}-\mathrm{CH}_{3}\right), 189(30, \\
\left.M^{\oplus}-\mathrm{NH}_{3}\right), 164(67, \\
\left.\mathrm{M}^{\oplus}-\mathrm{C}_{3} \mathrm{H}_{6}\right), 149(100, \\
\mathrm{M}^{\oplus}-\mathrm{C}_{4} \mathrm{H}_{9} 121(54, \\
149-\mathrm{CO}, 164-\mathrm{HNCO}), \\
78\left(17, \mathrm{C}_{5} \mathrm{H}_{4} \mathrm{~N}^{\oplus}\right), 51(13, \\
78-\mathrm{HCN}^{\top}, 44\left(19, \mathrm{H}_{2} \mathrm{NCO}^{\oplus}\right)\end{array}$ \\
\hline 5-(3,3-Dimethylbutanoyl)- . . & $\begin{array}{l}3420 \mathrm{~m}, 3170 \mathrm{~m} \\
2950 \mathrm{~m}, 1670 \mathrm{~s} \\
1585 \mathrm{w}\end{array}$ & $\begin{array}{l}1.08\left[\mathrm{~s}, \mathrm{C}\left(\mathrm{CH}_{3}\right)_{3}\right], 2.79(\mathrm{~s}, \\
\left.-\mathrm{CH}_{2}-\right), 5.78 \text { und } 7.76(\mathrm{~s}, \\
\left.\text { breit, } \mathrm{NH}_{2}\right), 8.25(\mathrm{dd}, \mathrm{H}-3) \\
8.31(\mathrm{dd}, \mathrm{H}-4), 9.08(\mathrm{~m}, \\
\mathrm{H}-6)\end{array}$ & $\begin{array}{l}29.14(3 \mathrm{C}), 30.68 \\
{\left[\mathrm{C}\left(\mathrm{CH}_{3}\right)_{3}\right], 49.90} \\
\left(\mathrm{CO}-\mathrm{CH} \mathrm{H}_{2}^{-}\right), 121.27 \\
134.48,136.00,138.47 \\
147.50,151.68,164.91 \\
\left(\mathrm{CONH}_{2}\right), 197.81(\mathrm{CO})^{\mathrm{d})}\end{array}$ & - \\
\hline $\begin{array}{l}\text { 5-(4-Trifluormethyl- } \\
\text { benzoyl)-... }\end{array}$ & $\begin{array}{l}3460 \mathrm{~m}, 3200 \mathrm{~m} \\
1685 \mathrm{~s}, 1660 \mathrm{~s} \\
1595 \mathrm{~m}\end{array}$ & $\begin{array}{l}7.83\left(\mathrm{mc}, \mathrm{AA}^{\prime} \mathrm{BB}^{\prime}-\mathrm{Typ}, 4 \mathrm{H}\right) \\
8.21(\mathrm{dd}, \mathrm{H}-4), 8.28(\mathrm{dd} \\
\mathrm{H}-3), 8.90(\mathrm{~m}, \mathrm{H}-6)^{\mathrm{c})}\end{array}$ & $\begin{array}{l}122.42,126.08\left(\mathrm{q}, J_{\mathrm{CF}}=\right. \\
4 \mathrm{~Hz}, 2 \mathrm{C}), 130.47(2 \mathrm{C}) \\
134.85,138.97,149.77 \\
169.07,197.31(\mathrm{CO})^{\mathrm{c})}\end{array}$ & $\begin{array}{l}294\left(69 \%, \mathrm{M}^{\oplus}\right), 275(8, \\
\left.\mathrm{M}^{\oplus}-\mathrm{F}\right), 251(100, \\
\left.\mathrm{M}^{\oplus}-\mathrm{HNCO}\right), 225(16, \\
\left.\mathrm{M}^{\oplus} \mathrm{CF}_{3}\right), 173(54, \\
\left.\mathrm{CF}_{3} \mathrm{C}_{6} \mathrm{H}_{4} \mathrm{CO}^{\oplus}\right), 145(59, \\
\left.\mathrm{CF}_{3} \mathrm{C}_{6} \mathrm{H}_{4}^{\oplus}\right), 121(8, \\
\left.\mathrm{M}^{\oplus}-173\right), 78(24 \\
\left.\mathrm{C}_{5} \mathrm{H}_{4} \mathrm{~N}^{\oplus}\right), 44\left(25, \mathrm{H}_{2} \mathrm{NCO}^{\oplus}\right)\end{array}$ \\
\hline 5-(Trifluormethylbenzoyl)-... & $\begin{array}{l}3440 \mathrm{~m}, 3200 \mathrm{~m} \\
1710 \mathrm{~s}, 1690 \mathrm{~s} \\
1655 \mathrm{~m}, 1610 \mathrm{w} \\
1595 \mathrm{~m}\end{array}$ & $\begin{array}{l}5.86(\mathrm{~s}, \text { breil, } \mathrm{NH}), 7.63 \\
\left(\mathrm{t}, J=8 \mathrm{~Hz}, \mathrm{H}-5^{\prime}\right), 7.80 \\
(\mathrm{~s}, \text { breit, NH), } 7.87 \text { und } \\
7.92\left(\mathrm{~d}, J=8 \mathrm{~Hz}, \mathrm{H}-4^{\prime} \text { und }\right. \\
\left.\mathrm{H}-6^{\prime}\right), 8.03\left(\mathrm{~s}, \mathrm{H}-2^{\prime}\right), 8.19 \\
(\mathrm{dd}, \mathrm{H}-4), 8.32(\mathrm{dd}, \mathrm{H}-3) \\
8.89(\mathrm{~m}, \mathrm{H}-6)\end{array}$ & $\begin{array}{l}\text { 119.90,124.05, 124.25, } \\
127.56,127.78,131.40, \\
132.13,135.16,136.48, \\
147.19,151.06,163.34 \\
\left.\left(\mathrm{CONH}_{2}\right), 190.69(\mathrm{CO})^{\mathrm{d}}\right)\end{array}$ & $\begin{array}{l}294\left(55 \%, \mathrm{M}^{\oplus}\right), 275(7, \\
\left.\mathrm{M}^{\oplus}-\mathrm{F}\right), 251(100, \\
\left.\mathrm{M}^{\oplus}-\mathrm{HNCO}\right), 225(14, \\
\left.\mathrm{M}^{\oplus}-\mathrm{CF}_{3}\right), 173(74, \\
\left.\mathrm{CF}_{3} \mathrm{C}_{6} \mathrm{H}_{4} \mathrm{CO}^{\oplus}\right), 145(89, \\
\left.\mathrm{CF}_{3} \mathrm{C}_{6} \mathrm{H}_{4}^{\oplus}\right), 121(18, \\
\left.\mathrm{M}^{\oplus}-173\right), 78(66, \\
\left.\mathrm{C}_{5} \mathrm{H}_{4} \mathrm{~N}^{\oplus}\right), 44\left(37, \mathrm{H}_{2} \mathrm{NCO}^{\oplus}\right)\end{array}$ \\
\hline
\end{tabular}


Tab. 7 (Fortsetzung)

\begin{tabular}{|c|c|c|c|c|}
\hline$\ldots$-2-pyridincarboxamid & $\begin{array}{c}\text { IR }(\mathrm{KBr})^{\mathrm{a})} \\
{\left[\mathrm{cm}^{-1}\right]}\end{array}$ & $\begin{array}{c}{ }^{1} \mathrm{H}-\mathrm{NMR}\left(\mathrm{CDCl}_{3}\right)^{\mathrm{b})} \\
\delta \text {-Werte }\end{array}$ & $\begin{array}{c}{ }^{13} \mathrm{C}-\mathrm{NMR}\left(\mathrm{CDCl}_{3}\right) \\
\delta \text {-Werte }\end{array}$ & $\begin{array}{c}\mathrm{MS}(70 \mathrm{eV}) \\
m / e\end{array}$ \\
\hline 5-(3-Cyanpropanoyl)-... & $\begin{array}{l}3400 \mathrm{~m}, 3200 \mathrm{~m}, \\
2260 \mathrm{w}, 1700 \mathrm{~s} \\
1665 \mathrm{~s}, 1600 \mathrm{~m}\end{array}$ & $\begin{array}{l}2.85(\mathrm{t}, J=8 \mathrm{~Hz}, \\
\left.-\mathrm{CH}_{2}-\right), 3.65(\mathrm{t}, J= \\
\left.8 \mathrm{~Hz},-\mathrm{CH} \mathrm{H}_{2}\right), 7.91(\mathrm{~s}, \text { breit, } \\
\mathrm{NH}), 8.24(\mathrm{~d}, \mathrm{H}-3), 8.31(\mathrm{~s}, \\
\text { breit, NH), } 8.56(\mathrm{dd}, \mathrm{H}-4), \\
9.22(\mathrm{~d}, \mathrm{H}-6)\end{array}$ & - & $\begin{array}{l}203\left(20 \%, \mathrm{M}^{\oplus}\right), 186(1, \\
\left.\mathrm{M}^{\oplus}-\mathrm{NH}_{3}\right), 160(61, \\
\mathrm{M}^{\oplus}-\mathrm{HNCO}^{\oplus}, 149(100, \\
\left.\mathrm{M}^{\oplus}-\mathrm{C}_{2} \mathrm{H}_{4} \mathrm{CN}\right), 121(39, \\
\left.\mathrm{M}^{\oplus}-\mathrm{COC}_{2} \mathrm{H}_{4} \mathrm{CN}\right), 106 \\
\left(19,149-\mathrm{HNCO}_{1}, 78(46,\right. \\
\left.\mathrm{C}_{5} \mathrm{H}_{4} \mathrm{~N}^{\oplus}\right), 44(30, \\
\left.\mathrm{H}_{2} \mathrm{NCO}^{\oplus}\right)\end{array}$ \\
\hline 5-(3-Nitrobenzoyl)-... & $\begin{array}{l}3420 \mathrm{~m}, 1670 \mathrm{~s} \\
1655 \mathrm{~m}, 1610 \mathrm{w} \\
1590 \mathrm{w}\end{array}$ & $\begin{array}{l}7.52(\mathrm{~s}, \text { breit, NH), } 7.83 \\
\left(\mathrm{m}, \mathrm{H}-5^{\prime}\right), 7.97(\mathrm{~s}, \text { breit, } \\
\mathrm{NH}), 8.19\left(\mathrm{~m}, \mathrm{H}-4^{\prime}\right), 8.28 \\
(\mathrm{dd}, \mathrm{H}-4), 8.36(\mathrm{dd}, \mathrm{H}-3), \\
8.53\left(\mathrm{~m}, \mathrm{H}-6^{\prime}\right), 8.62(\mathrm{~m}, \\
\left.\mathrm{H}-2^{\prime}\right), 8.97(\mathrm{~m}, \mathrm{H}-6)^{\mathrm{d})}\end{array}$ & $\begin{array}{l}120.07,122.39,125.58 \\
128.37,131.89,133.47 \\
135.79,136.50,146.35 \\
147.30,151.15,163.40 \\
\left.\left(\mathrm{CONH}_{2}\right), 189.96(\mathrm{CO})^{\mathrm{d}}\right)\end{array}$ & $\begin{array}{l}271\left(12 \%, \mathrm{M}^{\oplus}\right), 254(28, \\
\left.\mathrm{M}^{\oplus}-\mathrm{OH}, \mathrm{M}^{\oplus}-\mathrm{NH}_{3}\right), \\
228\left(100, \mathrm{M}^{\oplus}-\mathrm{HNCO}^{\oplus},\right. \\
182\left(12,228-\mathrm{NO}_{2}\right), 150 \\
\left(34, \mathrm{NO}_{2}-\mathrm{C}_{6} \mathrm{H}_{4} \mathrm{CO}^{\oplus}\right), \\
104\left(23, \mathrm{C}_{6} \mathrm{H}_{4} \mathrm{CO}^{\oplus}\right), 78(33, \\
\left.\mathrm{C}_{5} \mathrm{H}_{4} \mathrm{~N}^{\oplus}\right), 44(33, \\
\left.\mathrm{H}_{2} \mathrm{NCO}^{\oplus}\right)\end{array}$ \\
\hline 5-Butanoyl-N,N-dimethyl-... & $\begin{array}{l}2960 \mathrm{~m}, 2940 \mathrm{~m}, \\
2870 \mathrm{~m}, 1680 \mathrm{~s} \\
1625 \mathrm{~s} \text { (breit), } \\
1590 \mathrm{~m}\end{array}$ & $\begin{array}{l}1.03\left(\mathrm{t}, J=7 \mathrm{~Hz},-\mathrm{CH}_{3}\right) \\
1.80(\mathrm{sext}, J=7 \mathrm{~Hz} \\
\left.-\mathrm{CH}_{2}-\mathrm{CH}_{3}\right), 3.00(\mathrm{t} \\
\left.J=7 \mathrm{~Hz}, \mathrm{CO}-\mathrm{CH}_{2}-\right) \\
3.10 \text { und } 3.17\left[\mathrm{~s}, \mathrm{~N}\left(\mathrm{CH}_{3}\right)_{2}\right] \\
7.73(\mathrm{dd}, \mathrm{H}-3), 8.34(\mathrm{dd} \\
\mathrm{H}-4), 9.15(\mathrm{dd}, \mathrm{H}-6)\end{array}$ & $\begin{array}{l}13.78\left(-\mathrm{CH}_{3}\right), 17.42 \\
\left(-\mathrm{CH}_{2}-\mathrm{CH}_{3}\right), 35.74 \\
38.93\left[\mathrm{~N}\left(\mathrm{CH}_{3}\right)_{2}\right], 40.94 \\
\left(-\mathrm{CO}-\mathrm{CH}_{2}\right), 123.55 \\
132.38,136.41,148.24 \\
157.80,168.06 \\
{\left[-\mathrm{CON}\left(\mathrm{CH}_{3}\right)_{2}\right]}\end{array}$ & $\begin{array}{l}220\left(35 \%, \mathrm{M}^{\oplus}\right), 177(6, \\
\left.\mathrm{M}^{\oplus}-\mathrm{C}_{2} \mathrm{H}_{5} \mathrm{~N}\right), 163(14, \\
\left.\mathrm{M}^{\oplus}-\mathrm{CH}_{3},-\mathrm{C}_{3} \mathrm{H}_{7}\right) \\
149\left(60, \mathrm{M}^{\oplus}-\mathrm{C}_{3} \mathrm{H}_{7} \mathrm{CO}\right) \\
106\left(9, \mathrm{C}_{5} \mathrm{H}_{4} \mathrm{NCO}^{\oplus}\right), 44 \\
\left(100, \mathrm{C}_{2} \mathrm{H}_{5} \mathrm{NH}^{\oplus}\right)\end{array}$ \\
\hline 5-Butanoyl- $N$-tert-butyl- $\ldots$ & $\begin{array}{l}3380 \mathrm{~m}, 2990 \mathrm{w}, \\
2965 \mathrm{w}, 2935 \mathrm{w}, \\
1690 \mathrm{~s}, 1665 \mathrm{~s}, \\
1520 \mathrm{~s}\end{array}$ & $\begin{array}{l}1.03\left(\mathrm{t}, J=7 \mathrm{~Hz},-\mathrm{CH}_{3}\right) \\
1.51\left(\mathrm{~s},-\mathrm{C}\left(\mathrm{CH}_{3}\right)_{3}\right), 1.81 \\
(\mathrm{sext}, J=7 \mathrm{~Hz}, \\
\left.-\mathrm{CH}_{2}-\mathrm{CH}_{3}\right), 3.00(\mathrm{t}, J= \\
\left.7 \mathrm{~Hz},-\mathrm{CO}-\mathrm{CH}_{2}-\right), 8.02 \\
(\mathrm{~s}, \mathrm{breit}, \mathrm{NH}), 8.27(\mathrm{dd}, \\
\mathrm{H}-3), 8.36(\mathrm{dd}, \mathrm{H}-4), 9.06 \\
(\mathrm{~m}, \mathrm{H}-6)\end{array}$ & - & $\begin{array}{l}248\left(32 \%, M^{\oplus}\right), 233(100 \\
\left.M^{\oplus}-\mathrm{CH}_{3}\right), 205(14 \\
\left.\mathrm{M}^{\oplus}-\mathrm{C}_{3} \mathrm{H}_{7}\right), 176(27 \\
\left.\mathrm{M}^{\oplus}-\mathrm{C}_{4} \mathrm{H}_{9} \mathrm{NH}\right) \\
148\left(41, \mathrm{M}^{\oplus}-\right. \\
\left.\mathrm{C}_{4} \mathrm{H}_{9} \mathrm{HNCO}\right)\end{array}$ \\
\hline
\end{tabular}


- ${ }^{1} \mathrm{H}-\mathrm{NMR}\left(\mathrm{CDCl}_{3}\right): \delta=7.30\left(\mathrm{~d}, J_{65}=5 \mathrm{~Hz}, \mathrm{H}-5\right), 8.60\left(\mathrm{~d}, J_{65}=5 \mathrm{~Hz}, \mathrm{H}-6\right), 8.69(\mathrm{~s}, \mathrm{H}-2)$. MS $\left.(70 \mathrm{eV}): m / e=248\left(5 \%, \mathrm{M}^{\oplus}\right), 149\left(100, \mathrm{M}^{\oplus}-\mathrm{C}_{4} \mathrm{H}_{9} \mathrm{NCO}\right)\right]$.

5-Butanoyl-N, $N^{\prime}$-di-tert-butyl-2,4-pyridin-dicarboxamid: Ausb. ca. $3 \%$, Schmp. $156^{\circ} \mathrm{C}$, farblose Nadeln aus Methylenchlorid/Tetrachlorkohlenstoff. - IR (KBr): 3380, 3300 (m, N-H), 2975, $2940(\mathrm{w}, \mathrm{C}-\mathrm{H}), 1695,1670,1645$ (s, C = O und Amid I), 1565, 1530 (s, Amid II und C-C) $\mathrm{cm}^{-1} .-{ }^{1} \mathrm{H}-\mathrm{NMR}\left(\mathrm{CDCl}_{3}\right): \delta=1.00(\mathrm{t}, J=7 \mathrm{~Hz}, 3 \mathrm{H}), 1.46,1.49(\mathrm{~s}$, je $9 \mathrm{H}), 1.77$ (sext, $J=$ $7 \mathrm{~Hz}, 2 \mathrm{H}$ ), 2.85 (t, $J=7 \mathrm{~Hz}, 2 \mathrm{H}), 6.19,7.95$ (s, breit, NH), 8.19 (s, H-3), 8.60 (s, H-6). ${ }^{13} \mathrm{C}-\mathrm{NMR}\left(\mathrm{CDCl}_{3}\right): 13.36,17.60,28.61,28.66,44.50,51.34,52.67,118.94,136.62,145.03$, $146.91,152.45,162.03,165.30,202.56$. - MS $(70 \mathrm{eV}): m / e=347\left(17 \%, \mathrm{M}^{\oplus}\right), 332\left(75, \mathrm{M}^{\oplus}-\right.$ $\left.\mathrm{CH}_{3}\right), 304\left(53, \mathrm{M}^{\oplus}-\mathrm{C}_{3} \mathrm{H}_{7}\right), 275\left(51, \mathrm{M}^{\oplus}-\mathrm{C}_{4} \mathrm{H}_{9} \mathrm{NH}\right), 248\left(100, \mathrm{M}^{\oplus}-\mathrm{C}_{4} \mathrm{H}_{9} \mathrm{NCO}\right)$.

$\mathrm{C}_{19} \mathrm{H}_{29} \mathrm{~N}_{3} \mathrm{O}_{3}$ (347.5) $\quad$ Ber. C 65.68 H 8.41 N 12.09 Gef. C 65.57 H 8.39 N 12.21

Tab. 8. Ausbeuten und analytische Daten der in 5-Stellung substituierten 2-Pyridincarbonsäuren 5

\begin{tabular}{|c|c|c|c|c|c|c|c|}
\hline \multirow{2}{*}{ Substanz } & \multirow{2}{*}{ Ausb. a) } & \multirow{2}{*}{ Schmp. b) } & \multirow{2}{*}{$\begin{array}{l}\text { Summenformel } \\
\text { (Molmasse) }\end{array}$} & & \multicolumn{3}{|c|}{ Analyse } \\
\hline & & & & & & & $\mathrm{N}$ \\
\hline $\begin{array}{l}\text { 5-Benzyl-2-pyridin- } \\
\text { carbonsäurec) }^{\text {c }}\end{array}$ & 75 & 143 & $\begin{array}{l}\mathrm{C}_{13} \mathrm{H}_{11} \mathrm{NO}_{2} \\
(213.2)\end{array}$ & $\begin{array}{l}\text { Ber. } \\
\text { Gef. }\end{array}$ & $\begin{array}{l}73.22 \\
73.21\end{array}$ & $\begin{array}{l}5.20 \\
5.17\end{array}$ & $\begin{array}{l}6.57 \\
6.52\end{array}$ \\
\hline $\begin{array}{l}\text { 5-(4-Chlorbenzyl)-2- } \\
\text { pyridincarbonsäurec) }\end{array}$ & 92 & 151 & $\begin{array}{l}\mathrm{C}_{13} \mathrm{H}_{10} \mathrm{ClNO}_{2} \\
(247.7)\end{array}$ & $\begin{array}{l}\text { Ber. } \\
\text { Gef. }\end{array}$ & $\begin{array}{l}63.06 \\
63.31\end{array}$ & $\begin{array}{l}4.07 \\
4.05\end{array}$ & $\begin{array}{l}5.65 \\
5.66\end{array}$ \\
\hline $\begin{array}{l}\text { 5-(4-Methoxybenzyl)- } \\
\text { 2-pyridincarbon- } \\
\text { säurec) }\end{array}$ & 53 & 147 & $\begin{array}{l}\mathrm{C}_{14} \mathrm{H}_{13} \mathrm{NO}_{3} \\
(243.3)\end{array}$ & $\begin{array}{l}\text { Ber. } \\
\text { Gef. }\end{array}$ & $\begin{array}{l}69.12 \\
69.13\end{array}$ & $\begin{array}{l}5.39 \\
5.42\end{array}$ & $\begin{array}{l}5.76 \\
5.85\end{array}$ \\
\hline $\begin{array}{l}\text { 5-(4-tert-Butylbenzyl)- } \\
\text { 2-pyridincarbon- } \\
\text { säurec) }\end{array}$ & 83 & 190 & $\begin{array}{l}\mathrm{C}_{17} \mathrm{H}_{19} \mathrm{NO}_{2} \\
(269.3)\end{array}$ & $\begin{array}{l}\text { Ber. } \\
\text { Gef. }\end{array}$ & $\begin{array}{l}75.81 \\
76.10\end{array}$ & $\begin{array}{l}7.11 \\
7.13\end{array}$ & $\begin{array}{l}5.20 \\
5.20\end{array}$ \\
\hline $\begin{array}{l}\text { 5-Butyl-2-pyridin- } \\
\text { carbonsäurec, d) }\end{array}$ & 65 & $103-104^{e)}$ & - & - & & & \\
\hline $\begin{array}{l}\text { 5-(4-Trifluormethyl- } \\
\text { benzyl)-2-pyridin- } \\
\text { carboxamid c, f) }\end{array}$ & 45 & $196-200$ & $\begin{array}{l}\mathrm{C}_{14} \mathrm{H}_{11} \mathrm{~F}_{3} \mathrm{~N}_{2} \mathrm{O} \\
(280.2)\end{array}$ & $\begin{array}{l}\text { Ber. } \\
\text { Gef. }\end{array}$ & $\begin{array}{l}60.00 \\
60.28\end{array}$ & $\begin{array}{l}3.96 \\
4.26\end{array}$ & $\begin{array}{l}10.00 \\
10.68\end{array}$ \\
\hline $\begin{array}{l}\text { 5-(3-Trifluormethyl- } \\
\text { benzyl)-2-pyridin- } \\
\text { carbonsäure }\end{array}$ & 68 & 144.5 & $\begin{array}{l}\left.\mathrm{C}_{14} \mathrm{H}_{11} \mathrm{~F}_{3} \mathrm{~N}_{1.5} \mathrm{O}_{2} \mathrm{~g}\right) \\
\quad(289.3)\end{array}$ & $\begin{array}{l}\text { Ber. } \\
\text { Gef. }\end{array}$ & $\begin{array}{l}58.14 \\
58.99\end{array}$ & $\begin{array}{l}3.83 \\
3.71\end{array}$ & $\begin{array}{l}7.26 \\
7.53\end{array}$ \\
\hline $\begin{array}{l}\text { 4-(2-Carboxy-5-pyridyl)- } \\
\text { butansäurec) }\end{array}$ & 65 & $\begin{array}{l}158 \\
\text { (Zers.) }\end{array}$ & $\begin{array}{l}\left.\mathrm{C}_{10} \mathrm{H}_{13} \mathrm{~N}_{2} \mathrm{O}_{4}{ }^{h}\right) \\
(225.2)\end{array}$ & $\begin{array}{l}\text { Ber. } \\
\text { Gef. }\end{array}$ & $\begin{array}{l}53.32 \\
52.66\end{array}$ & $\begin{array}{l}5.82 \\
5.84\end{array}$ & $\begin{array}{l}12.44 \\
12.25\end{array}$ \\
\hline $\begin{array}{l}\text { 5-(4-Nitrobenzyl)- } \\
\text { 2-pyridincarbon- } \\
\text { säurei) }\end{array}$ & 90 & 179 & $\begin{array}{l}\mathrm{C}_{13} \mathrm{H}_{10} \mathrm{~N}_{2} \mathrm{O}_{4} \\
\quad(258.2)\end{array}$ & $\begin{array}{l}\text { Ber. } \\
\text { Gef. }\end{array}$ & $\begin{array}{l}60.47 \\
60.36\end{array}$ & $\begin{array}{l}3.90 \\
4.01\end{array}$ & $\begin{array}{l}10.85 \\
10.82\end{array}$ \\
\hline $\begin{array}{l}\text { 5-(3-Nitrobenzoyl)- } \\
\text { 2-pyridincarbon- } \\
\text { säurek) }\end{array}$ & 85 & $\begin{array}{l}177 \\
\text { (Zers.) }\end{array}$ & $\begin{array}{l}\left.\mathrm{C}_{14} \mathrm{H}_{12} \mathrm{~N}_{2} \mathrm{O}_{6}{ }^{\mathrm{l}}\right) \\
\quad(304.3)\end{array}$ & $\begin{array}{l}\text { Ber. } \\
\text { Gef. }\end{array}$ & $\begin{array}{l}55.27 \\
55.91\end{array}$ & $\begin{array}{l}3.98 \\
3.99\end{array}$ & $\begin{array}{l}9.21 \\
9.24\end{array}$ \\
\hline
\end{tabular}

a) Ausbeute in \% . - b) In ${ }^{\circ} \mathrm{C}$. - c) Durch Wolff-Kishner-Reduktion, s. o. - d) Aus 5-Butanoyl-2pyridincarboxamid und aus 5-Butanoyl- $N, N$-dimethyl-2-pyridincarboxamid mit vergleichbarer Ausb. - e) Lit. ${ }^{10)} 98^{\circ} \mathrm{C}$. - I) Bei der Umsetzung von 5-(4-Trifluormethylbenzoyl)-2-pyridincarboxamid ist die Reduktion der Ketogruppe bereits nach etwa 30-40 min vollständig, während die Amidgruppe unter diesen Bedingungen erhalten bleibt; zur Isolierung dieses Produktes wird bei der Aufarbeitung auf das Ansäuern verzichtet. - g) Kristalle enthalten 0.25 mol Hydrazin pro mol Säure. - h) Kristalle enthalten 0.5 mol Hydrazin pro mol Säure. - i) Durch Nitrierung von 5-Benzyl-2-pyridincarbonsäure. - k) Durch Umsetzung von 5-Benzoyl-2-pyridincarboxamid mit Nitriersäure. - I) Die Verbindung enthält $1 \mathrm{~mol}$ Kristall-Methanol (NMR-spektroskopischer Nachweis).

Liebigs Ann. Chem. 1982 
Tab. 9. Spektroskopische Daten der in 5-Stellung substituierten 2-Pyridincarbonsäuren 5

\begin{tabular}{|c|c|c|c|c|}
\hline Substanz & $\begin{array}{l}\operatorname{IR}(\mathrm{KBr}) \\
{\left[\mathrm{cm}^{-1}\right]}\end{array}$ & $\begin{array}{c}{ }^{1} \mathrm{H}-\mathrm{NMR}\left(\mathrm{CDCl}_{3}\right)^{\mathrm{a})} \\
\text {-Werte }\end{array}$ & $\begin{array}{c}{ }^{13} \mathrm{C}-\mathrm{NMR}\left(\mathrm{CDCl}_{3}\right) \\
\delta \text {-Werte }\end{array}$ & $\begin{array}{c}\mathrm{MS}(70 \mathrm{eV}) \\
m / e\end{array}$ \\
\hline 5-Benzyl-2-pyridincarbonsäure & $\begin{array}{l}3440 \mathrm{w}, \text { (breit), } \\
3020 \mathrm{~m}, 2405 \mathrm{~s}, \\
1890 \mathrm{~s}, 1700 \mathrm{~s}, \\
1690 \mathrm{~m}, 1675 \mathrm{~s}\end{array}$ & $\begin{array}{l}4.11\left(\mathrm{~s},-\mathrm{CH}_{2}-\right) \\
7.15-7.40(\mathrm{~m}, 5 \mathrm{H}), 7.73 \\
(\mathrm{dd}, \mathrm{H}-4), 8.17(\mathrm{~d}, \mathrm{H}-3) \\
8.68(\mathrm{~m}, \mathrm{H}-6)\end{array}$ & $\begin{array}{l}39.00\left(-\mathrm{CH}_{2}-\right), 124.38, \\
126.95,128.94(4 \mathrm{C}), 138.52, \\
138.68,141.66,145.15 \\
148.40,165.04\left(\mathrm{CO}_{2} \mathrm{H}\right)\end{array}$ & $\begin{array}{l}213\left(25 \%, \mathrm{M}^{\oplus}\right), 169(100, \\
\left.\mathrm{M}^{\oplus}-\mathrm{CO}_{2}\right), 91\left(28, \mathrm{C}_{7} \mathrm{H}_{7}^{\oplus}\right)\end{array}$ \\
\hline $\begin{array}{l}\text { 5-(4-Chlorbenzyl)-2-pyridin- } \\
\text { carbonsäure }\end{array}$ & $\begin{array}{l}3420 \mathrm{~m}, 3300 \mathrm{~m} \\
3160 \mathrm{~m}, 1710 \mathrm{~m} \\
1600 \mathrm{~s}, 1560 \mathrm{~s}\end{array}$ & $\begin{array}{l}4.00\left(\mathrm{~s},-\mathrm{CH}_{2}-\right), 7.20(\mathrm{mc} \\
\left.\mathrm{AA}^{\prime} \mathrm{BB}^{\prime}-\mathrm{Typ}, 4 \mathrm{H}\right), 7.70 \\
(\mathrm{dd}, \mathrm{H}-4), 8.16(\mathrm{~d}, \mathrm{H}-3) \\
8.61(\mathrm{~m}, \mathrm{H}-6)\end{array}$ & $\begin{array}{l}38.38\left(-\mathrm{CH}_{2}-\right), 124.28 \\
129.16(2 \mathrm{C}), 130.28(2 \mathrm{C}) \\
133.01,136.97,138.61 \\
141.18,145.21,148.45 \\
164.66\left(\mathrm{CO}_{2} \mathrm{H}\right)\end{array}$ & $\begin{array}{l}247\left(45 \%, \mathrm{M}^{\oplus}\right), 212(6, \\
\left.\mathrm{M}^{\oplus}-\mathrm{Cl}\right), 203(100 \\
\left.\mathrm{M}^{\oplus}-\mathrm{CO}_{2}\right), 167(39 \\
203-\mathrm{Cl}), 125(20 \\
\left.\left.\mathrm{C}_{7} \mathrm{H}_{6} \mathrm{Cl}\right)^{\oplus}\right)\end{array}$ \\
\hline $\begin{array}{l}\text { 5-(4-Methoxybenzyl)-2-pyridin- } \\
\text { carbonsäure }\end{array}$ & $\begin{array}{l}3440 \mathrm{~m} \text { (breit), } \\
3020 \mathrm{w}, 2930 \mathrm{~m}, \\
2840 \mathrm{w}, 2450 \mathrm{~m}, \\
1740 \mathrm{~m}, 1690 \mathrm{~m}, \\
1610 \mathrm{~m}, 1590 \mathrm{~m}, \\
1580 \mathrm{~m}\end{array}$ & $\begin{array}{l}3.74\left(\mathrm{~s}, \mathrm{OCH}_{3}\right), 3.98(\mathrm{~s}, \\
\left.-\mathrm{CH}_{2}-\right), 6.92(\mathrm{mc}, \\
\left.\mathrm{AA}^{\prime} \mathrm{BB}^{\prime}-\mathrm{Typ}, 4 \mathrm{H}\right), 7.63(\mathrm{dd}, \\
\mathrm{H}-4), 8.08(\mathrm{~d}, \mathrm{H}-3), 8.47(\mathrm{~m}, \\
\mathrm{H}-6)\end{array}$ & $\begin{array}{l}38.17\left(-\mathrm{CH}_{2}-\right), 55.31 \\
\left(\mathrm{OCH}_{3}\right), 114.44(2 \mathrm{C}), \\
124.16,129.95(2 \mathrm{C}), 130.55 \\
138.50,148.10,148.39 \\
158.63,166.90\left(\mathrm{CO}_{2} \mathrm{H}\right)\end{array}$ & $\begin{array}{l}243\left(50 \%, \mathrm{M}^{\oplus}\right), 213(1, \\
\left.\mathrm{M}^{\oplus}-\mathrm{CH}_{2} \mathrm{O}\right), 199(100 \\
\left.\mathrm{M}^{\oplus}-\mathrm{CO}_{2}\right), 184(4, \\
\left.199-\mathrm{CH}_{3}\right), 121(20, \\
\left.\mathrm{C}_{7} \mathrm{H}_{6} \mathrm{OCH}_{3}^{\oplus}\right), 91\left(4, \mathrm{C}_{7} \mathrm{H}_{7}^{\oplus}\right)\end{array}$ \\
\hline $\begin{array}{l}\text { 5-(4-tert-Butylbenzyl)-2-pyri- } \\
\text { dincarbonsäure }\end{array}$ & $\begin{array}{l}3440 \mathrm{~m} \text { (breit), } \\
3110 \mathrm{~m}, 2960 \mathrm{~s}, \\
2870 \mathrm{~m}, 2460 \mathrm{~m}, \\
1870 \mathrm{w}, 1700 \mathrm{~s}, \\
1590 \mathrm{~m}, 1580 \mathrm{~s}\end{array}$ & $\begin{array}{l}1.31\left[\mathrm{~s}, \mathrm{C}\left(\mathrm{CH}_{3}\right)_{3}\right], 4.06(\mathrm{~s}, \\
\left.-\mathrm{CH}_{2}-\right), 7.22(\mathrm{mc}, \\
\left.\mathrm{AA}^{\prime} \mathrm{BB}^{\prime}-\mathrm{Typ}, 4 \mathrm{H}\right), 7.73(\mathrm{dd}, \\
\mathrm{H}-4), 8.14(\mathrm{~d}, \mathrm{H}-3), 8.58(\mathrm{~m}, \\
\mathrm{H}-6)\end{array}$ & $\begin{array}{l}31.36\left[\mathrm{C}\left(\mathrm{CH}_{3}\right)_{3}\right], 34.50 \\
{\left[\mathrm{C}\left(\mathrm{CH}_{3}\right)_{3}\right], 38.62} \\
\left(-\mathrm{CH}_{2}-\right), 123.69,125.93 \\
(2 \mathrm{C}), 128.57(2 \mathrm{C}), 135.41 \\
138.58,142.08,144.41 \\
148.56,150.05,164.27 \\
\left(\mathrm{CO}_{2} \mathrm{H}\right)\end{array}$ & $\begin{array}{l}269\left(24 \%, \mathrm{M}^{\oplus}\right), 254(100, \\
\left.\mathrm{M}^{\oplus}-\mathrm{CH}_{3}\right), 225(5 \\
\left.\mathrm{M}^{\oplus}-\mathrm{CO}_{2}\right), 210(88 \\
\left.254-\mathrm{CO}_{2}\right), 91(20 \\
\left.\mathrm{C}_{7} \mathrm{H}_{7}^{\oplus}\right), 57\left(4, \mathrm{C}_{4} \mathrm{H}_{9}^{\oplus}\right)\end{array}$ \\
\hline 5-Butyl-2-pyridincarbonsäure & $\begin{array}{l}2950 \mathrm{~m}, 2850 \mathrm{w} \\
1750 \mathrm{w}, 1695 \mathrm{~m}\end{array}$ & $\begin{array}{l}0.94(\mathrm{t}, J=7 \mathrm{~Hz}, \\
\left.-\mathrm{CH}_{2}-\mathrm{CH}_{3}\right), 1.13-1.86 \\
\left(\mathrm{~m},-\mathrm{CH}_{2}-\mathrm{CH}_{2}-\right), 2.78(\mathrm{t}, \\
\left.J=7 \mathrm{~Hz}, \text { Pyridin- } \mathrm{CH}_{2}-\right) \\
7.83(\mathrm{dd}, \mathrm{H}-4), 8.24(\mathrm{~m}, \\
\mathrm{H}-3), 8.83(\mathrm{~m}, \mathrm{H}-6), 12.75 \\
\left(\mathrm{~s}, \mathrm{CO}_{2} \mathrm{H}\right)\end{array}$ & $\begin{array}{l}13.78\left(-\mathrm{CH}_{3}\right), 22.29 \\
\left(-\mathrm{CH}_{2}-\mathrm{CH}_{3}\right), 32.82 \\
\left(-\mathrm{CH}_{2}-\mathrm{CH}_{2}-\mathrm{CH}_{3}\right), 32.95 \\
\left(\mathrm{Pyr} .-\mathrm{C} \mathrm{H}_{2}-\right), 124.78, \\
138.56,143.04,145.45, \\
147.85,165.70\left(\mathrm{CO}_{2} \mathrm{H}\right)\end{array}$ & $\begin{array}{l}179\left(3 \%, \mathrm{M}^{\oplus}\right), 135(100, \\
\left.\mathrm{M}^{\oplus}-\mathrm{CO}_{2}\right), 92(18, \\
\left.\mathrm{C}_{6} \mathrm{H}_{6} \mathrm{~N}^{\oplus}\right)\end{array}$ \\
\hline $\begin{array}{l}\text { 5-(4-Trifluormethylbenzyl)- } \\
\text { 2-pyridincarboxamid }\end{array}$ & $\begin{array}{l}3420 \mathrm{~m}, 3300 \mathrm{~m} \\
3250 \mathrm{~m}, 2930 \mathrm{w} \\
1685 \mathrm{~s}, 1665 \mathrm{~s}, \\
1625 \mathrm{~m}, 1600 \mathrm{~m} \\
1575 \mathrm{~m}\end{array}$ & $\begin{array}{l}4.10\left(\mathrm{~s},-\mathrm{CH}_{2}-\right), 7.43(\mathrm{mc} \\
\left.\mathrm{AA}^{\prime} \mathrm{BB}^{\prime}-\mathrm{Typ}, 4 \mathrm{H}\right), 7.62(\mathrm{dd}, \\
\mathrm{H}-4), 8.11(\mathrm{~d}, \mathrm{H}-3), 8.42 \\
(\mathrm{~m}, \mathrm{H}-6), 6.50 \text { und } 7.85 \\
\left(\mathrm{~s}, \text { breit, } \mathrm{NH}_{2}\right)\end{array}$ & - & - \\
\hline
\end{tabular}


Tab. 9 (Fortsetzung)

\begin{tabular}{|c|c|c|c|c|}
\hline Substanz & $\underset{\left[\mathrm{cm}^{-1}\right]}{\operatorname{IR}(\mathrm{KBr})}$ & $\begin{array}{c}{ }^{1} \mathrm{H}-\mathrm{NMR}\left(\mathrm{CDCl}_{3}\right)^{\mathrm{a})} \\
\delta \text {-Werte }\end{array}$ & $\begin{array}{c}{ }^{13} \mathrm{C} \text {-NMR }\left(\mathrm{CDCl}_{3}\right) \\
\delta \text {-Werte }\end{array}$ & $\begin{array}{c}\mathrm{MS}(70 \mathrm{eV}) \\
m / e\end{array}$ \\
\hline $\begin{array}{l}\text { 5-(3-Trifluormethylbenzyl)- } \\
\text { 2-pyridincarbonsäure }\end{array}$ & $\begin{array}{l}3420 \mathrm{w}, 2940 \mathrm{~m}, \\
2900 \mathrm{~m}, 1730 \mathrm{~m}, \\
1590 \mathrm{~m}, 1565 \mathrm{~s}\end{array}$ & $\begin{array}{l}4.05\left(\mathrm{~s},-\mathrm{CH}_{2}-\right), \\
7.25-7.46(\mathrm{~m}, 4 \mathrm{H}), 7.55 \\
(\mathrm{dd}, \mathrm{H}-4), 7.99(\mathrm{~d}, \mathrm{H}-3), \\
8.49(\mathrm{~m}, \mathrm{H}-6)^{\mathrm{b})}\end{array}$ & $\begin{array}{l}38.34\left(-\mathrm{CH}_{2}-\right), 123.39 \\
\left.\text { (quart, } J_{\mathrm{CF}}=4 \mathrm{~Hz}\right), 124.70 \\
\left.125.33 \text { (quart, } J_{\mathrm{CF}}=4 \mathrm{~Hz}\right) \\
129.38,132.48,137.37 \\
139.04,140.35,149.55 \\
\left.154.90,166.60\left(\mathrm{CO}_{2} \mathrm{H}\right)^{\mathrm{b}}\right)\end{array}$ & $\begin{array}{l}281\left(8 \%, M^{\oplus}\right), 262(4, \\
\left.M^{\oplus}-F\right), 237(100, \\
\left.M^{\oplus}-C_{2}\right), 167(15), \\
159\left(12, C_{7} H_{6} C_{3}^{\oplus}\right)\end{array}$ \\
\hline $\begin{array}{l}\text { 4-(2-Carboxy-5-pyridyl)butan- } \\
\text { säure }\end{array}$ & $\begin{array}{l}2960 \mathrm{w}, 2920 \mathrm{w}, \\
1700 \mathrm{w}, 1595 \mathrm{~m}, \\
1565 \mathrm{~m}\end{array}$ & $\begin{array}{l}1.85 \text { (quint, } J=7 \mathrm{~Hz}, \\
-\mathrm{CH}_{2}-\mathrm{CH}_{2}-\mathrm{CH}_{2}-\text { ), } \\
2.20(\mathrm{t}, J=7 \mathrm{~Hz} \\
\left.-\mathrm{CH}_{2}-\mathrm{CO}_{2} \mathrm{H}\right), 2.70(\mathrm{t}, \\
\left.J=7 \mathrm{~Hz}, \text { Pyridin- } \mathrm{CH}_{2}-\right) \\
7.72(\mathrm{~d}, \mathrm{H}-4), 7.97(\mathrm{~d}, \\
\mathrm{H}-3), 8.48(\mathrm{~m}, \mathrm{H}-6)^{\mathrm{b})}\end{array}$ & - & - \\
\hline $\begin{array}{l}\text { 5-(4-Nitrobenzyl)-2-pyridin- } \\
\text { carbonsäure }\end{array}$ & $\begin{array}{l}3460 \mathrm{~m}, 3070 \mathrm{w} \\
1705 \mathrm{~m}, 1685 \mathrm{~s} \\
1595 \mathrm{~m}, 1510 \mathrm{~s}\end{array}$ & $\begin{array}{l}4.23\left(\mathrm{~s},-\mathrm{CH}_{2}-\right), 7.74(\mathrm{~d} \\
\mathrm{H}-4), 7.80\left(\mathrm{mc}^{-} \mathrm{AA}^{\prime} \mathrm{BB}^{\prime}-\mathrm{Typ}\right. \\
4 \mathrm{H}), 8.12(\mathrm{~d}, \mathrm{H}-3), 8.58 \\
(\mathrm{~m}, \mathrm{H}-6)^{\mathrm{c})}\end{array}$ & $\begin{array}{l}36.91\left(-\mathrm{CH}_{2}-\right), 122.42 \\
(2 \mathrm{C}), 123.52,128.23(2 \mathrm{C}) \\
136.42,137.74,144.86, \\
145.06,145.37,147.81 \\
164.58\left(\mathrm{CO}_{2} \mathrm{H}\right)^{\mathrm{c})}\end{array}$ & - \\
\hline $\begin{array}{l}\text { 5-(3-Nitrobenzoyl)-2-pyridin- } \\
\text { carbonsäure }\end{array}$ & $\begin{array}{l}3440 \mathrm{~m}, 3080 \mathrm{~m}, \\
2490 \mathrm{~m}, 1935 \mathrm{w}, \\
1690 \mathrm{~s}, 1670 \mathrm{~s} \\
1610 \mathrm{~m}, 1590 \mathrm{~m}\end{array}$ & $\begin{array}{l}7.82\left(\mathrm{~m}, \mathrm{H}-5^{\prime}\right), 8.19(\mathrm{~m}, \\
\left.\mathrm{H}-6^{\prime}\right), 8.33(\mathrm{~m}, \mathrm{H}-3 \text { und } \\
\mathrm{H}-4), 8.54\left(\mathrm{~m}, \mathrm{H}-4^{\prime}\right), 8.65 \\
\left(\mathrm{~m}, \mathrm{H}-2^{\prime}\right), 9.06(\mathrm{~m}, \mathrm{H}-6)^{\mathrm{c})}\end{array}$ & $\begin{array}{l}124.19,124.44,127.50 \\
130.53,134.01,135.69 \\
137.46,138.49,148.00 \\
149.99,151.51,105.50 \\
\left.\left(\mathrm{CO}_{2} \mathrm{H}\right), 191.98(\mathrm{CO})^{\mathrm{c}}\right)\end{array}$ & \\
\hline
\end{tabular}

a) Alle Spektren werden nach 1. Ordnung interpretiert; ungefähre Kopplungskonstanten für Pyridinprotonen: $J_{43}=8 \mathrm{~Hz}, J_{64}=2 \mathrm{~Hz}$; die Signale von $\mathrm{H}-3$ und H-6 sind bei allen Verbindungen außer 5-(Trifluormethylbenzyl)-2-pyridincarboxamid verbreitert. - b) Zusatz von [D] $\mathrm{D}_{6} \mathrm{DMSO}$. - c) Zusatz von $\left[D_{4}\right]$ Methanol. 
5-(3-Nitrobenzoyl)-2-pyridincarbonsäure: $1.7 \mathrm{~g}(8.0 \mathrm{mmol})$ 5-Benzoyl-2-pyridincarboxamid werden portionsweise innerhalb von $15-20 \mathrm{~min}$ in eine gerührte, eisgekühlte Mischung aus $2.4 \mathrm{ml}$ rauchender $\mathrm{HNO}_{3}$ und $3.5 \mathrm{ml}$ konz. $\mathrm{H}_{2} \mathrm{SO}_{4}$ eingetragen. Nach beendeter Zugabe rührt man noch 30 min unter Eiskühlung und anschließend $4 \mathrm{~h}$ bei Raumtemp. Man gießt auf etwa $100 \mathrm{~g}$ Eis und macht die Mischung mit halbkonz. Natronlauge alkalisch. Nach Auflösung des in saurer Lösung abgeschiedenen Feststoffs stellt man den $\mathrm{pH}$-Wert durch Zusatz von Phosphorsäure und etwas festem Natriumdihydrogenphosphat auf 4.5 ein. Bei $4{ }^{\circ} \mathrm{C}$ fällt aus der Lösung ein gelber Feststoff aus, den man aus Methanol unter Zusatz von wenig Wasser umkristallisiert. Ausb., physikalische und spektroskopische Daten siehe Tab. 8 und 9.

5-(4-Nitrobenzyl)-2-pyridincarbonsäure: $1.75 \mathrm{~g}(8.20 \mathrm{mmol})$ 5-Benzyl-2-pyridincarbonsäure (s. u.) gibt man portionsweise unter Rühren zu einer eisgekühlten, frisch zubereiteten Nitriermischung aus $3.3 \mathrm{ml}$ konz. $\mathrm{HNO}_{3}$ und $4 \mathrm{ml}$ konz. $\mathrm{H}_{2} \mathrm{SO}_{4}$. Man rührt noch $4 \mathrm{~h}$ bei $0^{\circ} \mathrm{C}$, läßt auf Raumtemp. erwärmen und gießt die Mischung auf etwa $40 \mathrm{ml}$ Eis/Wasser. Der pH-Wert der wäßrigen Phase wird durch Zusatz von Dinatriumhydrogenphosphat und $2 \mathrm{~N} \mathrm{NaOH}$ auf 4.5 eingestellt. Der ausgefallene Feststoff wird abgesaugt, mit destilliertem Wasser gewaschen und aus Ethanol umkristallisiert. Ausb., physikalische und spektroskopische Daten siehe Tab. 8 und 9.

5-Alkyl-2-pyridincarbonsäuren 23): $20 \mathrm{mmol} 5$-Acyl-2-pyridincarboxamid in $25 \mathrm{ml}$ Triethylenglycol werden mit $2.24 \mathrm{~g}(40.0 \mathrm{mmol})$ Kaliumhydroxid und $4.29 \mathrm{~g}(68.7 \mathrm{mmol}) 51 \mathrm{proz}$. Hydrazinlösung versetzt und unter Rühren und unter Schutzgas $\left(\mathrm{N}_{2}\right) 1 \mathrm{~h}$ lang auf $110^{\circ} \mathrm{C}$ erhitzt. AnschlieBend steigert man die Temp. des Bades auf $190^{\circ} \mathrm{C}$ und hält sie dort so lange, bis die Stickstoffentwicklung beendet ist und die dunkelrote Färbung der Reaktionsmischung nach Orange bis Gelb umschlägt. In der Regel ist dies nach $0.5-3 \mathrm{~h}$ der Fall. Der pH-Wert der erkalteten Lösung wird nach Verdünnen mit ca. $30 \mathrm{ml}$ dest. Wasser (Chelatbildung!) durch Zusatz von Phosphorsäure und etwas Kaliumdihydrogenphosphat auf pH 4.5 eingestellt. Die ausgefällte 5-Alkyl-2-pyridincarbonsäure wird abgesaugt, mit Wasser gewaschen und gegebenenfalls aus Ethanol, Methanol oder Ethanol/Wasser-Mischungen umkristallisiert.

Zur Isolierung der 4-(2-Carboxy-5-pyridyl)butansäure wird die alkalische Lösung zur Entfernung von Triethylenglycol zunächst viermal mit je $50 \mathrm{ml}$ Ether ausgeschüttelt. Die Dicarbonsäure wird dann ebenfalls bei $\mathrm{pH} 4.5$ als Monohydraziniumsalz ausgefällt, gegebenenfalls muß die wäßrige Phase eingeengt werden. Ausb., physikalische Daten und spektroskopische Daten siehe Tab. 8 und 9.

1) R. J. Matta und G. F. Wooten, Clin. Pharmacol. and Ther. 14, 541 (1973).

2) H. Hidaka und K. Takeya, Nature (London) 239, 334 (1972).

3) G. Büyük, Monatsh. Chemie 108, 437 (1977).

4) N. Finch, T. R. Campbell, C. W. Gemenden, M. J. Antonaccio und H. J. Povalksi, J. Med. Chem. 21, 1269 (1978).

5) K. Miyano, T. Sakasai und H. Hamano, Chem. Pharm. Bull. 26, 2328 (1978) und dort zit. Lit.

6) A. N. Kost, P. B. Terentev und L. V. Moshentseva, J. Indian Chem. Soc. 45, 1109 (1968).

7) K. Miyano, T. Koshigoe, T. Sakasai und H. Hamano, Chem. Pharm. Bull. 26, 1465 (1978) und dort zit. Lit.

8) E. Hardegger und E. Nikles, Helv. Chim. Acta 40, 2428 (1957).

9) M. Carissimi, P. de Meglio, P. Gentili, S. Manzardo und F. Ravenna, Farmaco, Ed. Sci. 34, 1039 (1979).

10) $R$. Tschesche und W. Führer, Chem. Ber. 111, 3502 (1978).

11) R. A. Abramovitch und G. M. Singer in R. A. Abramovitch, Pyridine and its Derivatives 14, Suppl. 1, 1. Aufl. Wiley, New York 1974.

12) B. Emmert und E. Asendorf, Ber. Dtsch. Chem. Ges. 72, 1188 (1939).

13) R. A. Barnes in E. Klingsberg, Pyridine and its Derivatives 1, Kap. 1, Ed. E. Klingsberg, Interscience, New York 1960. 
14) K. Miyano, T. Sakasai und H. Hamano, Heterocycles 14, 755 (1980).

15) F. Minisci, Fortschr. Chem. Forsch. 62, 1 (1976).

16) F. Minisci und O. Porta, Adv. Heterocycl. Chem. 16, 123 (1974).

17) F. Minisci, Synthesis 1973, 1 .

18) E. Fritz, Diplomarbeit, Univ. Freiburg 1979.

19) H. H. Jaffé, Chem. Rev. 53, 191 (1953).

20) E. Fritz, H. Langhals und C. Rüchardt, Liebigs Ann. Chem. 1981, 1015.

21) Über die Minisci-Acylierung mit Oxalsäureperestern als Radikalquelle wird gesondert berichtet. E. Langhals, $H$. Langhals und $C$. Rüchardt, unveröffentlicht.

${ }^{22)}$ F. J. Villani und M. S. King in N. Rabjohn, Org. Synth. IV, S. 88, Wiley, New York 1963.

23) R. L. Frank und C. Weatherbee, J. Am. Chem. Soc. 70, 3482 (1948).

24) H. Stetter, H. Kuhlmann und G. Lorenz in R. M. Coates, Org. Synth. 59, 53 (1980).

25) C. G. Stuckwisch, G. G. Hammer und N. F. Blau, J. Org. Chem. 22, 1678 (1957).

26) A. Arnone, M. Cecere, R. Galli, F. Minisci, M. Perchinunno, O. Porta und G. Gardini, Gazz. Chim. Ital. 103, 13 (1973).

27) J. Inczedy, Analytical Application of Complex Equilibria, 1. Aufl., Wiley 1976.

28) T. M. Medvedeva, A. M. Bezborodov, M. D. Zheludkova, N. F. Zhdanova und A. N. Kudnin, Khim. Farm. Zh. 12, 66 (1978); übersetzt in: Pharm. Chem. J. 12, 614 (1978).

29) $R$. Wolffenstein und $F$. Hartwich, Ber. Dtsch. Chem. Ges. 48, 2043 (1915).

30) G. Cavallini, E. Milla, E. Grumelli, F. Ravenna und J. Grasso, Farmaco, Ed. Sci. 12, 853 (1957).

31) T. Fujii, T. Hiraga, S. Yoshifuji, M. Ohba und K. Yoshida, Chem. Pharm. Bull. 26, 3233 (1978).

32) Darstellung analog J. S. Meek und D. T. Osuga in H. E. Baumgarten, Org. Synth. V, S. 126, Wiley, New York 1973.

33) J. Moffat, M. V. Newton und G. J. Papenmeier, J. Org. Chem. 27, 4058 (1962).

$[180 / 81]$ 